\title{
Recent Advances on MOF Derivatives for Non-Noble Metal Oxygen Electrocatalysts in Zinc-Air Batteries
}

Cite as

Nano-Micro Lett.

(2021) 13:137

Received: 22 March 2021

Yuting Zhu ${ }^{1,3}$, Kaihang Yue ${ }^{1,3}$, Chenfeng Xia ${ }^{2}$, Shahid Zaman ${ }^{2}$, Huan Yang ${ }^{2}$, Xianying Wang ${ }^{3}$, Ya Yan ${ }^{1,3}$, Bao Yu Xia ${ }^{2}$

Accepted: 11 May 2021

Published online: 7 June 2021

(C) The Author(s) 2021

\section{HIGHLIGHTS}

- This review summarizes the recent progress and application of different metal-organic frameworks (MOFs)-derived non-noble metal materials for zinc-air batteries in the past few years.

- This work gives extensive insights in understanding the relationship between design strategies and structure-activity relationship.

- The challenges and prospects of MOF-derived oxygen electrocatalysts for zinc-air batteries are proposed.

\begin{abstract}
Oxygen electrocatalysts are of great importance for the air electrode in zinc-air batteries (ZABs). Owing to the high specific surface area, controllable pore size and unsaturated metal active sites, metal-organic frameworks (MOFs) derivatives have been widely studied as oxygen electrocatalysts in ZABs. To date, many strategies have been developed to generate efficient oxygen electrocatalysts from MOFs for improving the performance of ZABs. In this review, the latest progress of the MOF-derived non-noble metal-oxygen electrocatalysts in ZABs is reviewed. The performance of these MOF-derived catalysts toward oxygen reduction, and oxygen evolution reactions is discussed based on the categories of metal-free carbon materials, single-atom catalysts, metal cluster/carbon composites and metal compound/ carbon composites. Moreover, we provide a comprehensive overview on the design strategies of various MOF-derived non-noble metal-oxygen electrocatalysts and their structure-performance relationship. Finally, the challenges and perspectives are provided for further advancing the MOF-derived oxygen electrocatalysts in ZABs.
\end{abstract}

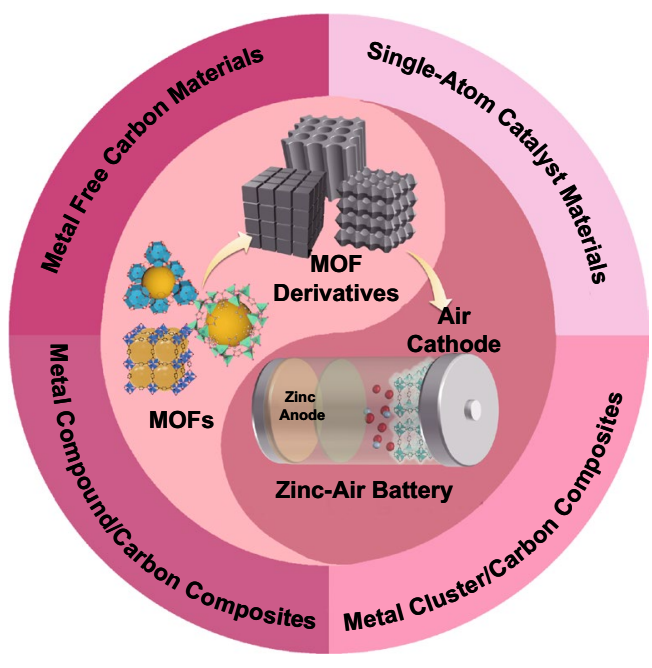

KEYWORDS Metal-organic framework; Non-noble metal; Oxygen electrocatalysts; Air electrode; Zinc-air batteries

Yuting Zhu and Kaihang Yue contributed equally to this work.

$\triangle$ Xianying Wang, wangxianying@mail.sic.ac.cn; Ya Yan, yanya@mail.sic.ac.cn; Bao Yu Xia, byxia@hust.edu.cn

1 School of Materials Science \& Engineering, University of Shanghai for Science and Technology, 516 Jungong Road, Shanghai 200093, People's Republic of China

2 Key Laboratory of Material Chemistry for Energy Conversion and Storage (Ministry of Education), Hubei Key Laboratory of Material Chemistry and Service Failure, Wuhan National Laboratory for Optoelectronics, School of Chemistry and Chemical Engineering, Huazhong University of Science and Technology (HUST), 1037 Luoyu Road, Wuhan 430074, People's Republic of China

3 CAS Key Laboratory of Materials for Energy Conversion, Shanghai Institute of Ceramics, Chinese Academy of Sciences (SICCAS), Shanghai 200050, People's Republic of China 


\section{Introduction}

Recent couple of decades has witnessed rapid technological development, which increased energy demands of the society; where the massive consumptions of conventional oil and gas resources have caused severe environmental problems [1]. Therefore, to eradicate the environmental issues, there is an urgent need to explore and develop new sustainable energy alternatives for the traditional non-renewable energy systems [2-4]. Hence, alternate electrochemical energy storage devices [5], such as fuel cells [6-11], lithium-ion batteries [12,13], solar cells [14, 15], supercapacitors [16-18] and metal-air batteries [19-26] have been widely explored. Among these renewable sources, zinc-air batteries have attracted great attention due to their high specific energy density, environmental friendliness and safety [27-29].
Zinc-air batteries possess second highest weight-specific energy after the Li-air batteries, while the volume-specific energy is the highest among the other renewable energy sources (Fig. 1a). Thus, zinc-air batteries are considered to be a promising system because of their richly available raw materials, low cost, mildness of zinc electrode in the reaction process and the non-flammable aqueous electrolytes [30-32].

A typical zinc-air battery is usually composed of a zinc electrode, air electrode, electrolyte and a separator. The catalyst on the air electrode is one of the most important components, which is mainly responsible for the oxygen electrochemical reactions including oxygen reduction reaction (ORR) and oxygen evolution reaction (OER). The activity and stability of oxygen electrocatalysts play a significant role in the discharging-charging performance of ZABs [33-35]. (a)

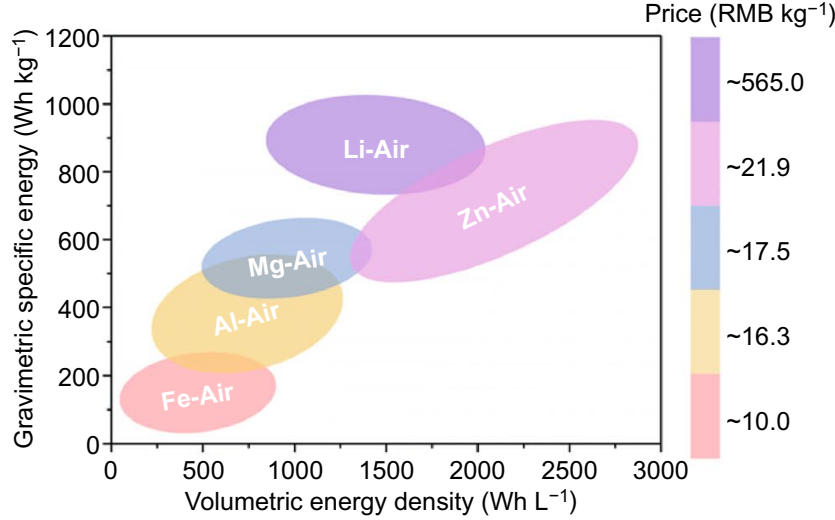

(c)

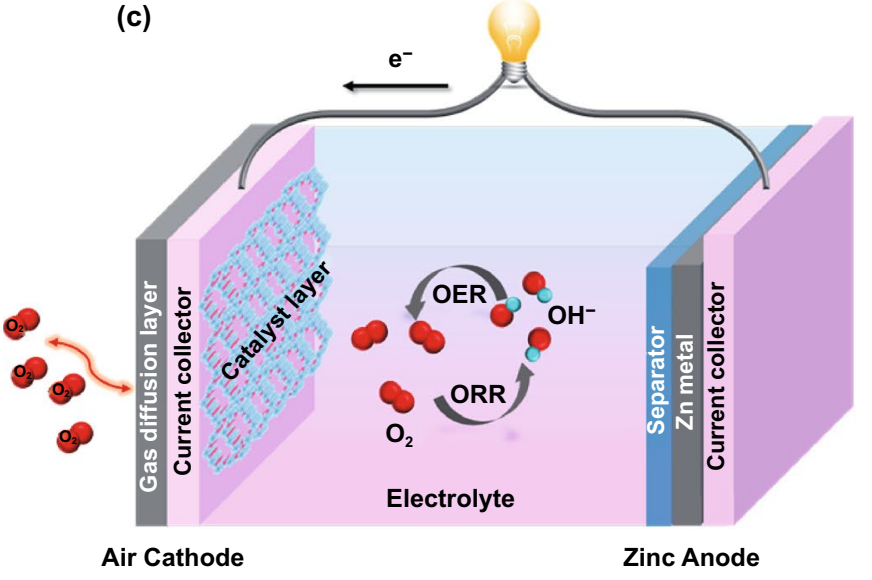

(b)
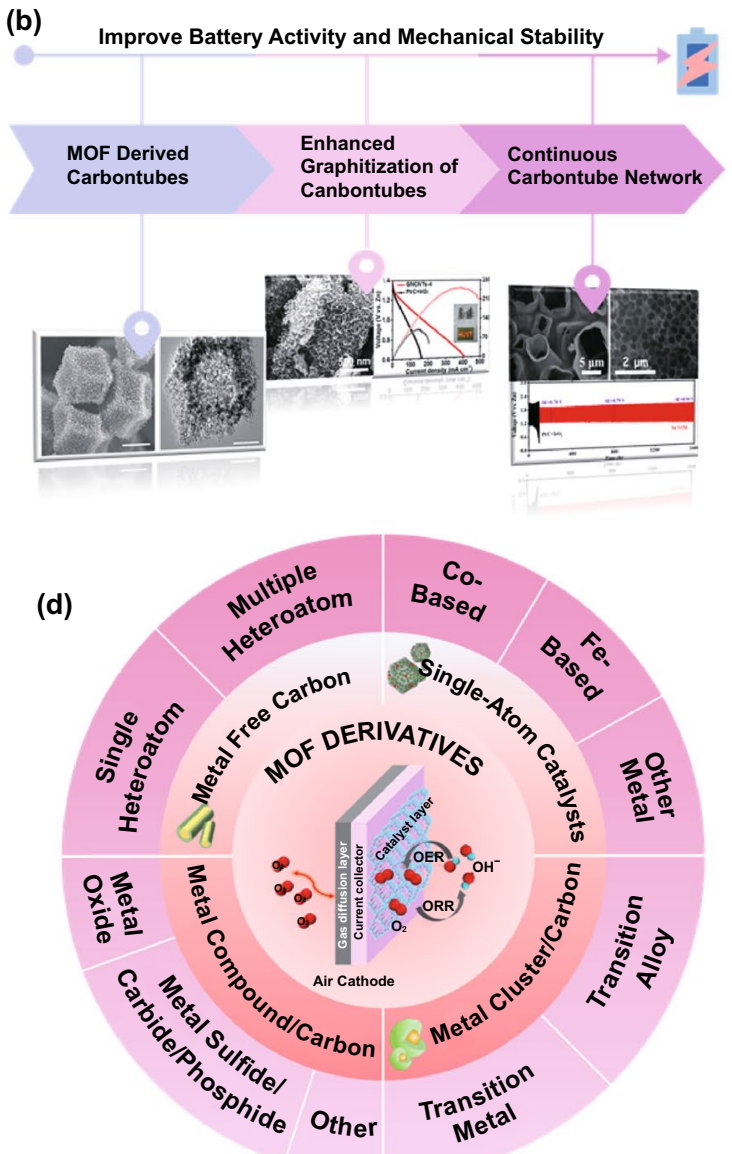

Fig. 1 a Comparison of gravimetric specific energy and volumetric energy density of several batteries. b Progress of our research in the development of MOF derivatives in ZABs. $\mathbf{c}$ Typical device of the ZABs and air electrode of ZABs structure. d Classification diagram of MOFderived catalysts for air cathode 
At present, the most active catalysts are the precious metalbased materials, such as ruthenium and platinum-based catalysts. The high costs and low reserves of these precious metals greatly hinder their widespread applications in zincair batteries. Therefore, the development of highly efficient electrocatalysts based on non-noble metal materials for air electrodes has become imperative to realize the commercialization of $\mathrm{ZABs}$.

Metal-organic frameworks (MOFs), also known as covalent organic frameworks (COFs) or coordination polymers, are crystalline porous materials with a periodic structure formed by the coordination of metal ions or clusters with organic ligands [36]. Up to now, MOFs have been applied to an ocean of fields, such as catalysis, energy storage, conversion, gas adsorption and separation [37]. Besides, as catalysts and catalytic host materials [38], MOFs are frequently used as a precursor to developing MOF-derived electrode catalysts, which not only retain the advantages of the original structure of MOFs but also provide enhanced conductivity and stability $[39,40]$. Based on the versatile and unique structure-activity characteristics of $\mathrm{MOF}$-derived nanomaterials toward oxygen electrocatalysis in ZABs, our group has contributed many leading, innovative and systematic works (Fig. 1b). We have reported a well-defined hollow structure composed of cobalt incorporated into nitrogen-doped carbon nanotubes by the thermal annealing of zeolitic-imidazolate frameworks (ZIFs) for the first time as electrocatalyst for ZABs [41]. This work provides a prospect for the development of highly active oxygen electrocatalysts in electrochemical energy devices [42-44]. To further enhance the activity and stability of MOF-derived carbon nanotubes for zinc-air batteries, the graphitization degree of carbon nanotubes can be improved from the enhanced carbon bonding at the micro-macro scale, to achieve the enhanced corrosion resistance, stability and conductivity of the electrocatalysts [45]. Thereafter, we focused on the design of both efficient and stable bifunctional oxygen electrocatalysts for long-life metal-air batteries [46-48]. A template method has been developed to prepare a variety of three-dimensional continuous carbon nanotube network materials for liquid and flexible solid-state zinc-air batteries to improve performance and cycle stability [49-51]. When applied to the rechargeable zinc-air batteries, strong cycle stability has been attempted up to $1600 \mathrm{~h}$. Therefore, with substantial contributions in the preparation of MOFs derived oxygen catalysts for zinc-air batteries, we anticipate to work on the design of
MOF-derived carbon-based catalysts, including optimizing the structure of carbon-based catalysts, improving the utilization of active metal atoms, improving the conductivity, stability, corrosion resistance of catalyst carriers, etc., to improve energy power density and cycling durability of ZABs.

There are many pieces of research on MOFs-based materials [52-54], however, most of them focus on the synthesis of MOFs-based catalysts for ORR or OER under different conditions, and little attention has been paid to the MOFderived electrocatalysts as air cathode in $\mathrm{ZABs}$, including ORR catalysts and OER/ORR bifunctional catalysts. Moreover, there is huge room to describe the relationship between catalyst material structures, oxygen electrocatalytic activity and the practical problems in the application of zinc-air batteries. In this review, the recent progress in MOF-derived oxygen electrocatalysts as air cathode in zinc-air batteries is comprehensively discussed and summarized with the focus on understanding the design strategies and the structure-activity relationship. Starting with a brief introduction to the fundamentals of oxygen electrolysis in ZABs, the recent advances on MOF-derived non-noble metal-oxygen electrocatalysts are successively reviewed for ORR and OER from the category of metal-free carbon materials, singleatom catalysts, transitional metal cluster/carbon composites and metal compound/carbon composites. In particular, these MOF-derived non-noble metal-oxygen electrocatalysts in the ZABs are reviewed based on the structure-performance relationship. Finally, the challenges and prospects of MOFderived non-noble-metal oxygen electrocatalysts in ZABs are proposed. We hope that this review and the provided references will play a guiding role to contribute in the development of MOF-derived oxygen electrocatalytic materials in ZABs.

\section{Oxygen Electrolysis in $\mathrm{ZABS}$}

\subsection{Architecture and Working Mechanism}

A typical configuration of zinc-air battery is usually composed of air cathode, zinc anode, electrolyte and separator (Fig. 1c) $[55,56]$. A multitude of the anode material of ZABs usually uses a gel mixture mixed with granular zinc powder or a pure $\mathrm{Zn}$ plate. The air electrode is the key technology of ZABs. The oxygen in the air is the active and inexhaustible substance of 
electrode reaction, which makes the air electrode have high specific energy compared with the oxide electrode as a cathode material of general batteries. Generally, the gas diffusion layer, the current collector and the catalyst layer form a sandwich structure, which composes of the traditional air electrode (Fig. 1c). The components of the air electrode include: (1) current collector, the current collector is nickel mesh made of nickel metal, nickel foam or a cheaper metal mesh with nickel coating [31]. (2) catalytic layer, this is the place where ORR occurs in the primary ZABs, or OER and ORR occur in the secondary ZABs, which is the key to the performance of the ZABs, (3) gas diffusion layer, the main function of the gas diffusion layer is to let the reaction gas pass through smoothly and transport the corresponding gas needed for the reaction active layer. At the same time, the gas diffusion layer must prevent the gas diffusion channel from being covered due to the flow of electrolyte, which requires the gas diffusion layer to have a highly limited surface area. For example, polytetrafluoroethylene (PTFE) is generally the main part of the gas diffusion layer and can be doped with other carbon materials to form the gas diffusion layer [57].

Usually, the well-known working mechanism of ZABs is the oxidation-reduction reaction between zinc anode and air cathode during the discharge and charge process [27]. During the discharge process, oxygen diffuses to the air electrode, where it is reduced to hydroxyl ions under the action of an active catalyst. The hydroxyl ions generated on the air cathode migrate to the zinc anode through the separator, and then combine with zinc ions to form soluble zincate ion $\left(\mathrm{Zn}(\mathrm{OH})_{4}{ }^{2-}\right)$, when the zincate ion in the electrolyte reaches saturation, it will decompose to $\mathrm{ZnO}$. In this process, the electrons released by the reaction between zinc and hydroxide are transferred to the air cathode through an external circuit, and the oxygen in the air in contact with the air cathode undergoes an ORR at the air cathode [58]. During the charging process, OER occurs at the air cathode, and the final reaction is the decomposition of $\mathrm{ZnO}$ into $\mathrm{Zn}$ and $\mathrm{O}_{2}$. The specific process is:

Air cathode: $\mathrm{O}_{2}+2 \mathrm{H}_{2} \mathrm{O}+4 \mathrm{e}^{-} \rightarrow 4 \mathrm{OH}^{-}$

Zinc anode $: \quad \mathrm{Zn} \rightarrow \mathrm{Zn}^{2+}+2 \mathrm{e}^{-}$

$\mathrm{Zn}^{2+}+2 \mathrm{OH}^{-} \rightarrow \mathrm{Zn}(\mathrm{OH})_{2}$

$\mathrm{Zn}(\mathrm{OH})_{2} \rightarrow \mathrm{ZnO}+\mathrm{H}_{2} \mathrm{O}$
Total reaction: $2 \mathrm{Zn}+\mathrm{O}_{2} \rightarrow 2 \mathrm{ZnO}$

The catalysts employed in the air electrode determine the efficiency of the ZABs, therefore, reasonable assessment should be established to evaluate the oxygen electrochemical catalysts from a single reaction to the whole battery architecture. Generally, the performance of the ORR catalyst is evaluated by comparing the reduction peak potential, onset potential, half-wave potential and stability, while the performance of the OER catalysts is usually estimated by the overpotential to achieve the current density of $10 \mathrm{~mA} \mathrm{~cm}^{-2}$ as well as its durability. During the charge-discharge process of ZABs, the OER and ORR occur on air electrode alternately. The challenge for the air electrode is the two different overpotentials needed to trigger the OER and ORR processes because the OER requires a larger overpotential to occur $(\approx 2.0 \mathrm{~V}$ or even higher), but at this voltage, the ORR catalyst will be deactivated during high-voltage charging. Therefore, the catalysts with good ORR activity generally show poor OER performance. For this reason, the development of nonprecious metal materials with both OER and ORR functional catalytic properties is crucial for the development of ZABs, especially rechargeable ZABs [59]. Besides, $6 \mathrm{M}$ $\mathrm{KOH}$ is frequently used as the electrolyte for the primary batteries, and $\mathrm{Zn}(\mathrm{AC})_{2}$ is usually added to the $6 \mathrm{M} \mathrm{KOH}$ electrolyte in the rechargeable batteries. To assess the performance of the constructed $\mathrm{ZABs}$, attention should be paid to open-circuit voltage (OCV), charging voltage $\left(\mathrm{V}_{\mathrm{C}}\right)$, discharging voltage $\left(\mathrm{V}_{\mathrm{D}}\right)$, peak power density (PPD), specific capacity, stability and other physical parameters. In light of the structure-activity relationship, the obtained performance results can be further used to optimize the design of the oxygen catalysts to achieve higher performance of ZABs [60].

\subsection{Oxygen Electrochemical Reactions in Air Electrode}

It is obvious that for both primary ZABs and rechargeable ZABs, the ORR is an essential electrochemical process, which is the key reaction of the ZABs. The ORR mechanism is also complicated due to the multiple-electron reaction process. At present, the normally accepted ORR 
mechanism involves a four-electron reaction mechanism and/or two-electron reaction mechanism [61]. In the ORR, the best-performing precious metal catalysts are mainly four-electron reactions. The process of both reaction mechanisms is explained as follows:

Four-electron reaction:

Acidic electrolyte: $\mathrm{O}_{2}+4 \mathrm{H}^{+}+4 \mathrm{e}^{-} \rightarrow 2 \mathrm{H}_{2} \mathrm{O}$

Alkaline electrolyte: $\mathrm{O}_{2}+2 \mathrm{H}_{2} \mathrm{O}+4 \mathrm{e}^{-} \rightarrow 4 \mathrm{OH}^{-}$

Two-electron reaction:

Acidic electrolyte: $\mathrm{O}_{2}+2 \mathrm{H}^{+}+2 \mathrm{e}^{-} \rightarrow \mathrm{H}_{2} \mathrm{O}_{2}$

$\mathrm{H}_{2} \mathrm{O}_{2}+2 \mathrm{H}^{+}+2 \mathrm{e}^{-} \rightarrow 2 \mathrm{H}_{2} \mathrm{O}$

Alkaline electrolyte: $\mathrm{O}_{2}+2 \mathrm{H}_{2} \mathrm{O}+4 \mathrm{e}^{-} \rightarrow \mathrm{HO}_{2}^{-}+\mathrm{OH}^{-}$

$\mathrm{HO}_{2}^{-}+\mathrm{H}_{2} \mathrm{O}+2 \mathrm{e}^{-} \rightarrow 3 \mathrm{OH}^{-}$

In the rechargeable ZABs, the oxygen electrocatalyst is also vital to catalyze the reverse reaction of ORR, that is OER [62-66]. When charging, the zinc compound will be formed at the anode, and the air cathode will release oxygen [67]. Analogous to ORR, OER also involves the transfer of four electrons, so its reaction mechanism is also very complicated. At present, there are two main points of view to explain the reaction mechanism of OER, namely: (1) directly combine two $\mathrm{M}-\mathrm{O}$ to generate $\mathrm{O}_{2}$; (2) first generate $\mathrm{M}-\mathrm{OOH}$, and then generate $\mathrm{O}_{2}$ to from $\mathrm{M}-\mathrm{OOH}$, here $\mathrm{M}$ means active site. Compared with the ORR, the OER kinetics of rechargeable ZABs are much slower, a charging voltage of about $2.0 \mathrm{~V}$ or higher is often required, and the open-circuit voltage of the discharging process is generally around $1.2 \mathrm{~V}$. Furthermore, the excessive charging voltage will cause the corrosion of air electrode and oxidation of the electrocatalyst [29]. Certainly, the existing three-electrode structure allows a rechargeable zincair battery to be assembled using two single functional oxygen electrocatalysts to achieve dual functions. The three-electrode system battery increases the volume and weight of the battery while improving the stability, which inevitably reduces the volume and mass-energy. So, the air cathode catalyst is preferred to be functional for both OER and ORR instead of the three-electrode system. This puts forward higher requirements for the development of highly active and stable OER catalysts, even more, efficient bifunctional catalysts for catalyzing both OER and ORR.

In general, the charge-discharge process of ZABs requires two oxygen electrochemical processes, OER and ORR, on the air cathode. However, due to intrinsic high kinetic barrier of OER and ORR, highly efficient electrocatalysts are required to meet the desired power output. Considering the diverse structural characteristics of MOFs derived electrocatalytic materials, the MOF-derived oxygen electrocatalysts provide more possibilities for performance optimization in ZABs. Particularly, the high specific surface area can lead to more accessible active sites in contact with electrolyte. When MOFs is used as a support matrix, its crystal framework can ensure the uniform distribution of active units, which avoids the formation of large agglomerates and provides a diversified platform for the synthesis of high-performance metals or carbon-based derivatives [68-70]. Therefore, MOFs and their derivatives are promising electrode materials for ZABs.

\section{MOF-Derived Oxygen Electrocatalysts as Air Electrode in ZABs}

MOFs are the organic-inorganic hybrid materials, which possess a variety of metal ions and organic ligands coordinated to form a variety of structures. Compared with the pristine MOFs, MOF-derived materials inherit the porous characteristics of original MOFs to a great extent, which realize the precise regulation of the active ingredients in the derived materials. We summarize the different types of MOF derivatives as oxygen electrocatalysts in ZABs (Fig. 1d). The following parts will review and discuss these advanced MOF-derived oxygen electrocatalysts successively from the category of metal-free carbon materials, single-atom catalysts, metal cluster/carbon composites and metal compound/ carbon composites.

\subsection{MOF-Derived Metal-Free Carbon Materials}

Carbon materials have the advantages of low cost, environmental acceptability, positive conductivity and stability $[71,72]$. When doped with heteroatom such as nitrogen, phosphorus, boron, sulfur, oxygen and other heteroatoms, 
they often exhibit enhanced catalytic activity. Compared with metal-based catalysts, MOF-derived metal-free carbon materials are promising alternative electrocatalysts for ORR and are frequently employed as air cathode catalysts in ZABs [73-76].

As a type of host materials, metal-free carbon materials derived from MOFs have also been studied as oxygen catalysts, especially the nitrogen-rich porous carbon (NPC) [77-79]. When used as cathode electrocatalysts in the ZABs, the doped $\mathrm{N}$ can terminate the electrical neutrality of adjacent carbon atoms and form a positively charged position due to the electron-withdrawing characteristics of $\mathrm{N}$ atoms. This character can promote oxygen adsorption and thus improve the cathode ORR performance. In a novel work by Yang et al. [80], an effective and universal silica-template strategy has been used to form an ordered macro-porous carbon skeleton with narrow connections/walls between the spherical voids (BHPC, Fig. 2a) by in-situ growing ZIF-8 crystal particles on the surface of the silica microspheres and subsequent carbonization to remove the template. Compared with NC-950 $\left(1331 \mathrm{~m}^{2} \mathrm{~g}^{-1}, 1.71 \mathrm{~cm}^{3} \mathrm{~g}^{-1}\right)$, BHPC-950 had a larger total pore volume of $13.42 \mathrm{~cm}^{3} \mathrm{~g}^{-1}$, a larger specific surface area of $2546 \mathrm{~cm}^{2} \mathrm{~g}^{-1}$, high $\mathrm{N}$ content of 7.6 at\% and ordered interconnected pore network, which highlighted the advantages of this dual template strategy. As shown in Fig. 2b, by using the BHPC-950 as an air electrode, the assembled ZABs could not only work at a high rate of $120 \mathrm{~mA} \mathrm{~cm}^{-2}$ but also provide an excellent capacity of $770 \mathrm{mAh} \mathrm{g}^{-1}$. Besides, two constructed ZABs connected in series could light up a light-emitting diode composed of 30 LEDs $(2.2 \mathrm{~V})$ for $12 \mathrm{~h}$ without brightness attenuation. These results indicated that the highly exposed graphite $\mathrm{N}$ under the double template had unique texture characteristics, which would promote the cathodic ORR in ZABs and could be used as a promising substitute for Pt-based catalysts in energy devices. Also, $\mathrm{ZnO}$ and $\mathrm{ZIF}-8$ composite were used as the sacrifice template to fabricate hollow N-doped carbon microspheres (Fig. 2c) [81]. The large pore volume and high surface area in the microspheres not only promote the diffusion of electrolyte and gas molecules but also offer richly accessible active sites. Moreover, the addition of glucose as extra carbon source to the ZIF could improve the graphitization degree of samples and help to remove zinc metal and zinc compound impurities, giving an effective route to synthesize metal-free nitrogen-doped porous carbon [82]. These ZIF-derived metal-free electrocatalysts usually exhibit excellent electrocatalytic activity and operational stability for ORR with a great potential in ZABs. Therefore, as an advanced platform, MOF-derived porous carbon will significantly broaden the family of nanoporous carbon materials with novel structure and versatile properties for oxygen electrolysis in ZABs.

Multi-nonmetallic heteroatom doping is another important strategy to utilize the mutual synergistic effect of different atoms to improve the oxygen electrocatalytic performance of the carbon materials [83, 84]. Qian et al. [85] reported B-N double-doped porous carbon (BNPC) for ORR/OER catalysis by thermal decomposition of Zn-MOF (MC-BIF-1S) in an $\mathrm{H}_{2}-\mathrm{Ar}$ mixed atmosphere, which decomposed into cracked BNPC solids with a porous structure (Fig. 2d). It was a good example that MOF material was used to prepare the metal-free bifunctional electrocatalyst. The larger pores in the catalyst could greatly reduce the mass transfer resistance. The cracking and porous structure of BNPC produced a sea of large pores, which could be used as channels for reactants to pass through the electrode layer, thereby improving catalytic performance. BNPC-1100 as an oxygen catalyst on the air cathode in rechargeable ZABs exhibited a charge potential of $2.19 \mathrm{~V}$ at a current density of $2 \mathrm{~mA} \mathrm{~cm}^{-2}$, and the discharge potential was $2.16 \mathrm{~V}$. The cycling stability of the assembled batteries was tested for $100 \mathrm{~h}$ without significant performance loss (Fig. 2e). Besides, $\mathrm{S}$ and $\mathrm{N}$ co-doped porous carbon was also synthesized by using urea as nitrogen source dimethyl sulfoxide as sulfur source in the MOF-5 template [86]. The synergistic effect of $\mathrm{N}$ and $\mathrm{S}$ in NS(3:1)-CMOF-5 as a metal-free electrocatalyst for ORR showed the highest initial potential, even comparable to the $\mathrm{Pt} / \mathrm{C}$ catalyst. These porous carbon materials derived from self-sacrificial MOFs templates have more unique structural characteristics than traditional carbon materials. Inheriting the advantages of MOFs, the pore structure becomes more variable to meet the demand, and the specific surface area is greatly improved, which will affect the final performance of the material. Therefore, by selecting desired dopants and proper MOF precursors, multi-nonmetallic heteroatom-doped carbon materials can be ingeniously designed as advanced oxygen electrocatalysts, which are very promising to be used as catalytic materials for air cathode in ZABs.

\subsection{MOF-Derived Single-Atom Catalysts}

Platinum/iridium-based catalysts are often benchmark electrocatalysts used to improve oxygen electrolysis in ZABs [87, 88], reducing the catalyst loading and increasing 
(a)

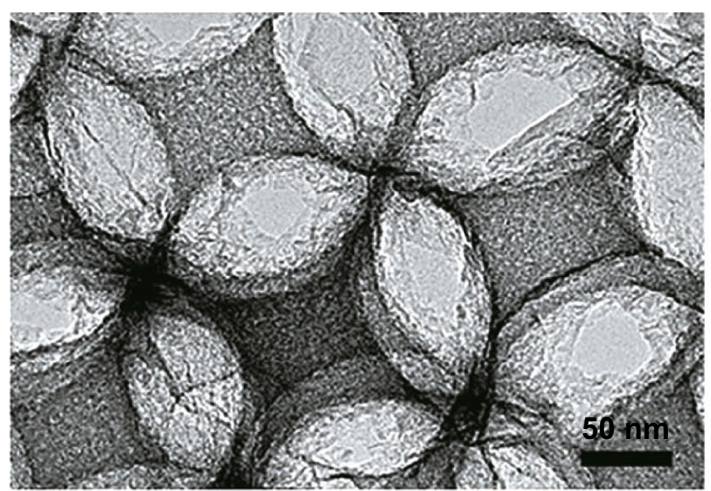

(b)

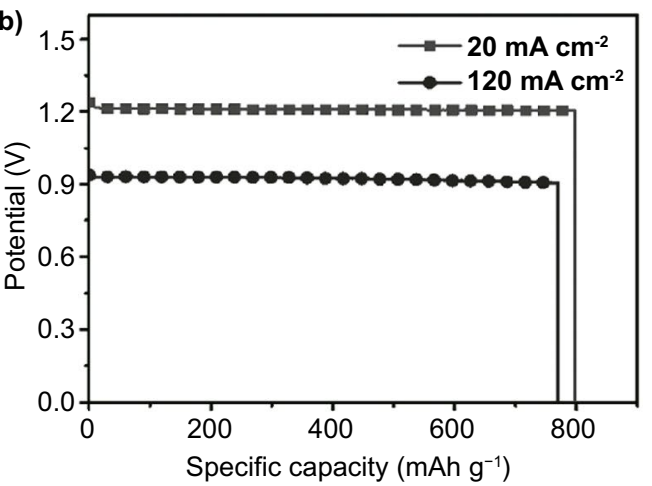

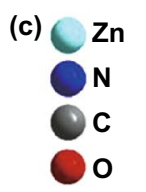
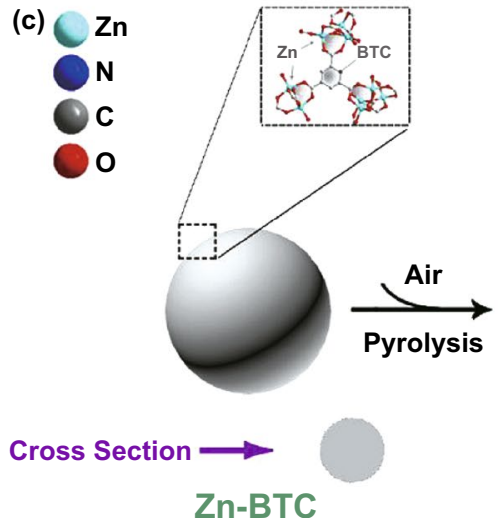

(d)

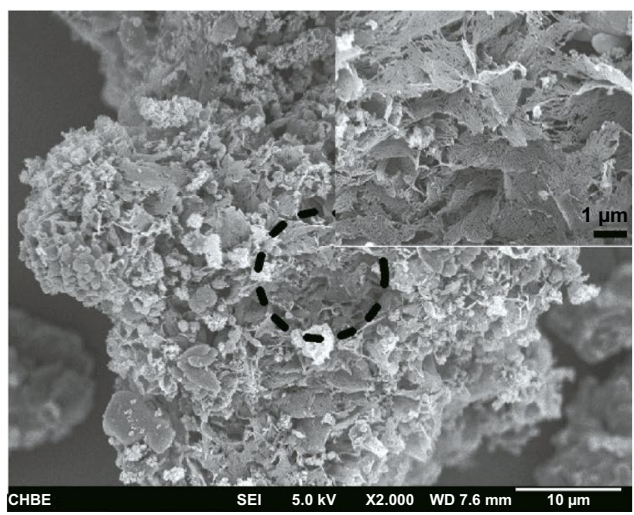

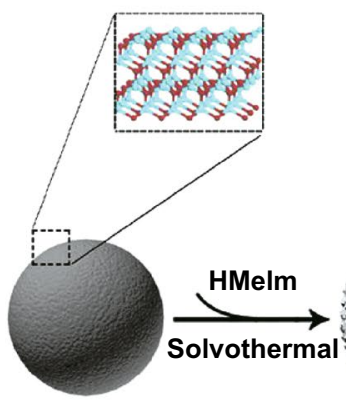
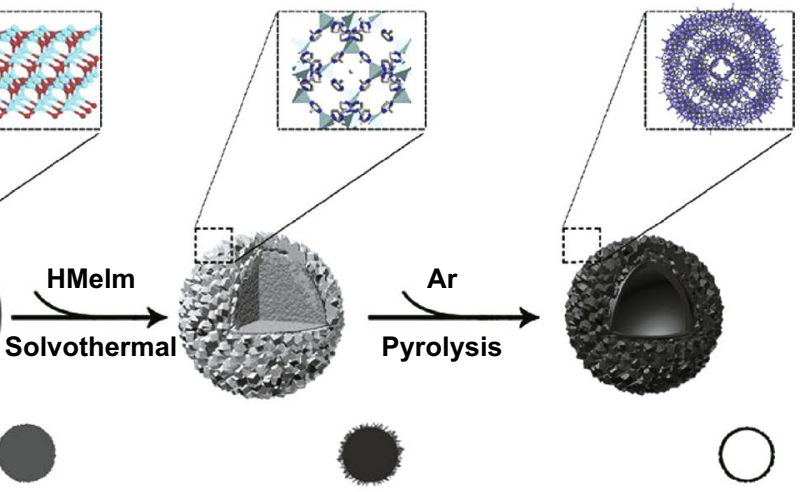

$\mathrm{ZnO}$

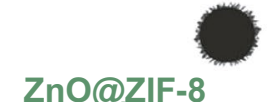

HNCSS

Time (hour)

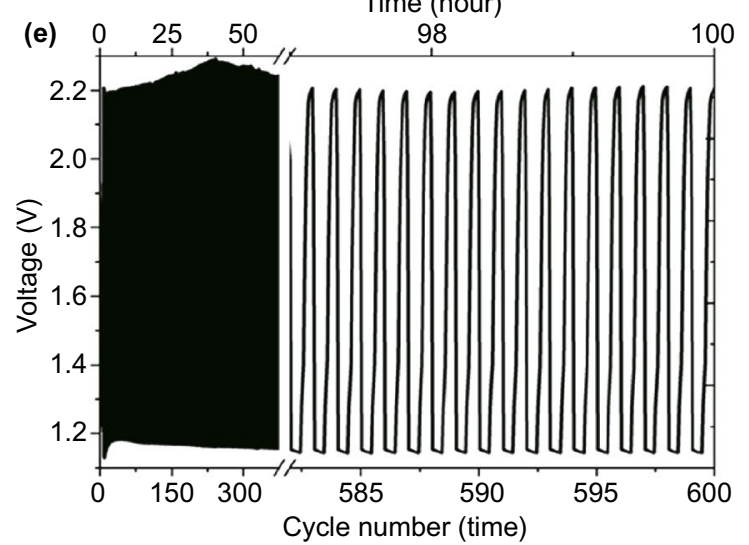

Fig. 2 a TEM images and b specific capacity for ZABs of MOF-derived nitrogen-rich carbon photonic crystal (BHPC-950). Copyright @ 2018 WILEY-VCH Verlag GmbH \& Co. KGaA, Weinheim. c Schematic preparation of hollow N-doped carbon spheres through in-situ growth and calcination strategy. Copyright @ 2019 Elsevier Ltd. d SEM image of BNPC-1100 and e cycling performance of the rechargeable Zn-air batteries using BNPC-1100 as the air cathode. Copyright @ 2016 Elsevier Ltd

intrinsic catalytic activity of these noble metal-based oxygen electrocatalysts are critical to realizing their application on large-scale. In this regard, single-atomic catalysts (SACs) are promising due to favorable active sites uniformity, high product selectivity, multiple support types, high atomic efficiency and low precious metal usage [89-91]. However, it is difficult and challenging to synthesize SACs because with the dispersion of catalysts at the atomic level, the surface free energy increase sharply leading to serious aggregation [92]. Owing to the unique structural characteristics, MOFs present an effective a template or host to design SACs [93]. Besides, the MOF-derived noble metal SACs, reports on 
MOF-derived non-precious metal containing SACs are also widely explored [94-99]. The following parts will focus on the application of MOF-derived transition metal (e.g., Co, $\mathrm{Fe}$ and $\mathrm{Mn}$ ) single-atom oxygen catalysts in ZABs.

\subsubsection{MOF-Derived Co-based SACs}

The nitrogen coordinated cobalt atom in the carbon matrix ( $\mathrm{Co}-\mathrm{N}-\mathrm{C})$ is considered to be the ideal material to replace the noble metal Pt-based materials for the ORR [100]. To improve the utilization, efficiency of active Co metal by designing the Co single-atom decorated $\mathrm{N}$-doped porous carbon materials is an effective way [101, 102]. For instance, Zang et al. [103] used a Co-MOF to prepare the CoSA electrocatalyst in $\mathrm{N}$-doped porous carbon nanosheets array by carbonization and acidification process. The obtained NCCoSA in a wafer array had well-dispersed Co single-atoms, connected to the carbon network through $\mathrm{Co}-\mathrm{N}$ bonds, because of the extra porosity in the carbon network and an active surface area generated by removing Co metal clusters. With Co nanoparticles grown on NC comparison, the NCCoSA electrocatalyst with a high-density of $\mathrm{Co}-\mathrm{N}_{x}$ active centers had the lower OER overpotential and higher ORR saturation current, which indicated that Co metal clusters were driving OER, and ORR aspect is superfluous. Therefore, the NC-CoSA electrocatalyst assembled on the carbon cloth used as the air cathode without binder, and additive was applied in the solid-state ZABs. The constructed batteries demonstrated positive cycle stability and high open-circuit potential. Unlike the simple carbonization and acidification method, Ji et al. [104] reported a novel impregnation carbonization acidification (ICA) method to prepare atom-dispersed $\mathrm{Co}-\mathrm{N}$ sites within the porous carbon sheet arrays grown on carbon nanofibers using ZIFs and electrospun nanofibers (ENFs) as precursors. During the synthesis process, ZIF-Ls evolved into N-doped carbon flakes, while cobalt ion nitrogen coordination units in ZIFs were reduced in-situ to form atom-dispersed $\mathrm{Co}-\mathrm{N}$ sites. The excess cobalt atoms aggregated and caused the formation of Co clusters, which were removed by an acid leaching process to form CoSA@NCF/CNF with outstanding flexibility. Therefore, the wearable ZABs composed of the CoSA@NCF/CNF air electrode had a high deformation tolerance and promising potential as an integrated batteries system.
In addition to above discussed single CoSACs, MOFderived Co-monatomic catalyst complexes also display excellent electrocatalytic properties. A representative example is hollow carbon nanotubes integrated with single Co atoms and $\mathrm{Co}_{9} \mathrm{~S}_{8}$ nanoparticles $\left(\mathrm{CoSA}+\mathrm{Co}_{9} \mathrm{~S}_{8} / \mathrm{HCNT}\right)$ prepared by the in-situ self-sacrificing method [105]. A $\mathrm{ZnS} / \mathrm{ZIF}-67$ hybrid was used as a template, after the carbonization treatment, the $\mathrm{ZnS}$ self-sacrificing nanorods are formed a tubular structure, which served as a sulfur source for the formation of $\mathrm{Co}_{9} \mathrm{~S}_{8}$ nanoparticles (Fig. 3a). The obtained $\mathrm{CoSA}+\mathrm{Co}_{9} \mathrm{~S}_{8} / \mathrm{HCNT}$ showed outstanding oxygen electrocatalytic activity, and its potential difference (the difference between $E_{j=10}$ and $E_{1 / 2}$ ) was $0.705 \mathrm{~V}$, much smaller than the potential difference of $\mathrm{Pt} / \mathrm{C}+\mathrm{RuO}_{2}$ $(0.777 \mathrm{~V})$. The peak power density of the liquid ZABs assembled with $\mathrm{CoSA}+\mathrm{Co}_{9} \mathrm{~S}_{8} / \mathrm{HCNT}$ reached 177.33 $\mathrm{mW} \mathrm{cm}{ }^{-2}$, much better than $\mathrm{Pt} / \mathrm{C}+\mathrm{RuO}_{2}$ (Fig. 3b). And at $1 \mathrm{~mA} \mathrm{~cm}{ }^{-2}$, the flexible $\mathrm{Zn}$-air batteries based on $\mathrm{CoSA}+\mathrm{Co}_{9} \mathrm{~S}_{8} / \mathrm{HCNT}$ catalyst still maintained a stable discharge/charge potential after a long cycle and had excellent durability (Fig. 3c).

\subsubsection{MOF-Derived Fe-Based SACs}

In parallel, various MOF-derived Fe-based single-atom catalysts are also widely studied. Previously, Wang and his coworkers reported a hollow structure composed of single iron atom sites on $\mathrm{N}, \mathrm{P}$ and $\mathrm{S}$ co-doped hollow carbon polyhedron (Fe-SAs/NPS-HC) constructed by MOF@polymer composite [106]. They highlighted that the polymerbased coating modulated the electrons of the active metal center through the Kirkendall effect while the close coordination of $\mathrm{N}$ and the long-distance interaction of $\mathrm{S}$ and $\mathrm{P}$ promoted the construction of hollow structures (Fig. 3d). Thus, the as-prepared Fe SAs/NPS-HC catalyst showed an excellent ORR performance in alkaline medium, with a half-wave potential $\left(E_{1 / 2}\right)$ of $0.912 \mathrm{~V}$, while in acidic media, a $E_{1 / 2}$ of $0.791 \mathrm{~V}$ was obtained, which was close to the catalytic performance of $\mathrm{Pt} / \mathrm{C}$ catalyst and better than the majority of non-noble metal catalysts. Density functional theory (DFT) calculations revealed that the dispersion of $\mathrm{N}$-coordinated $\mathrm{Fe}$ atoms and the electronic effects of surrounding $\mathrm{S}$ and $\mathrm{P}$ atoms are responsible for the efficient and satisfactory dynamics of Fe-SAs/NP-HC. 
(a)

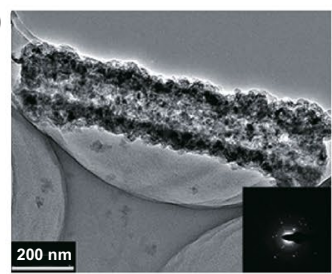

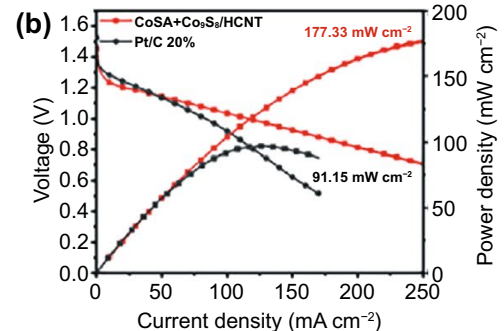

Current density $\left(\mathrm{mA} \mathrm{cm}^{-2}\right)$ (d)

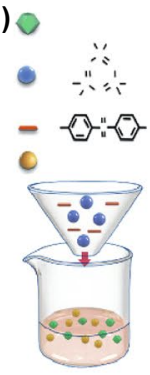

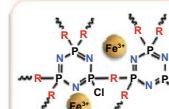

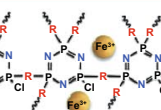

3 等,
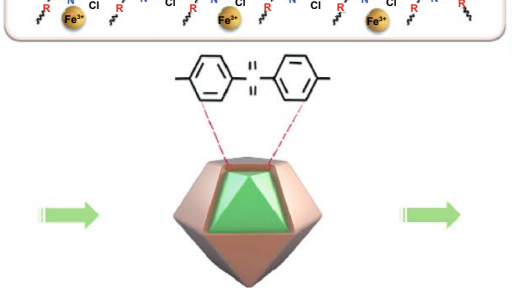

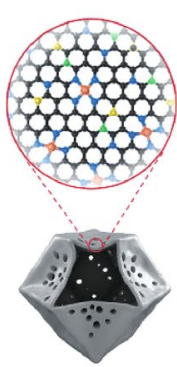

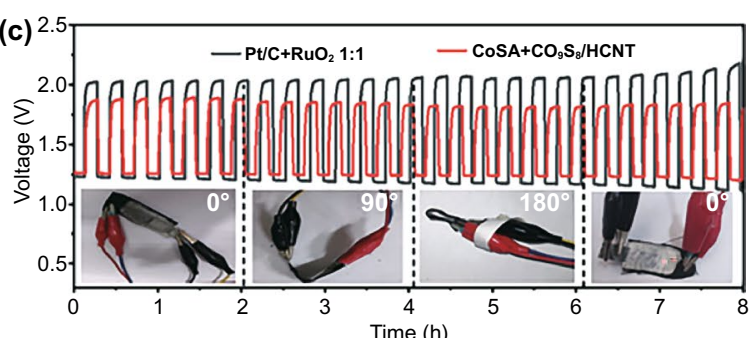

(e)

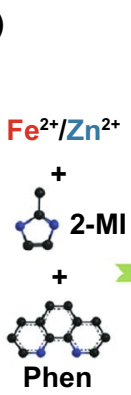

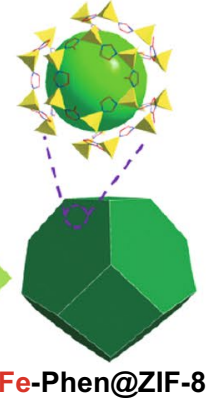

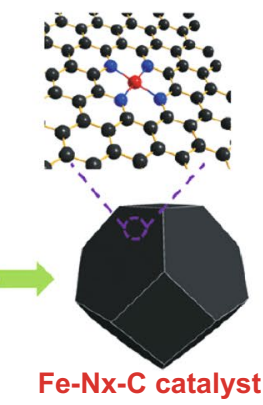

Time (s)

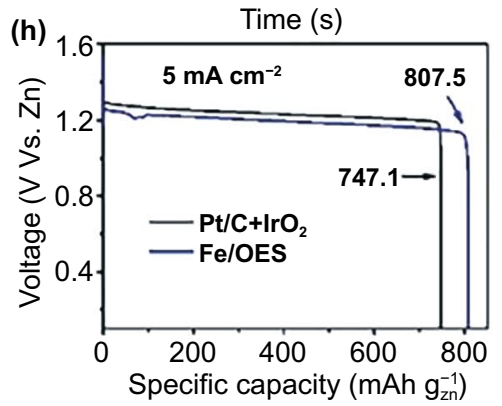

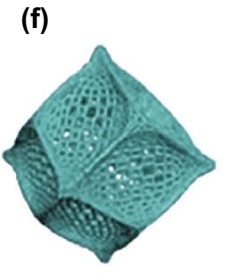

Fe/OES
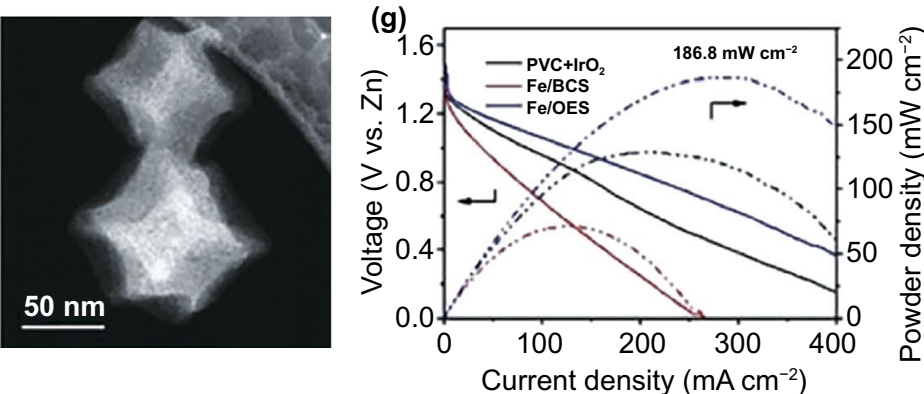

Fig. 3 a-c TEM image, power density curves and discharge-charge cycling profiles (solid-state) of $\mathrm{Zn}$-air batteries based on $\mathrm{CoSA}+\mathrm{Co}_{9} \mathrm{~S}_{8} /$ HCNT. Copyright (c) 2020 WILEY-VCH Verlag GmbH \& Co. KGaA, Weinheim. d Preparation of single iron atomic sites supported on nitrogen, phosphorus and sulfur co-doped hollow carbon polyhedron (Fe-SAs/NPS-HC). Copyright () 2018 Nature Publishing Group. e Synthesis diagram of $\mathrm{Fe}-\mathrm{N}_{x}-\mathrm{C}$ catalyst. Copyright $\odot 2019$ WILEY-VCH Verlag GmbH \& Co. KGaA, Weinheim. f-h SEM image, power density plots and the specific capacities of Fe/OES. Copyright @ 2020 Wiley-VCH Verlag GmbH \& Co. KGaA, Weinheim

The atomic Fe center provided electrons, which weakened the charge of $\mathrm{Fe}\left(\mathrm{Fe}^{\delta+}\right)$, thereby weakening the binding of $\mathrm{Fe}\left(\mathrm{Fe}^{\delta+}\right)$ charge to $\mathrm{OH}$ species. Moreover, the constructed ZABs indicated that Fe-SAs/NPS-HC had strong competitiveness compared with $\mathrm{Pt} / \mathrm{C}$, suggesting the prospects in energy storage and conversion devices.

In these MOF-derived Fe-based SACs, $\mathrm{Fe}-\mathrm{N}_{6}, \mathrm{Fe}-\mathrm{N}_{4}$ and $\mathrm{Fe}-\mathrm{N}_{2}$ coordination are often considered to be the main active components that affecting the oxygen electrocatalytic performance [107-109]. $\mathrm{Fe}-\mathrm{N}-\mathrm{C}$ is one of the most representative MOF-derived transition metal single-atom electrocatalyst material. Combination of experiments and DFT calculations, Han et al. [110] systematically studied the proximity effect of two adjacent $\mathrm{Fe}-\mathrm{N}-\mathrm{C}$ sites on ORR in a monodispersed $\mathrm{Fe}-\mathrm{N}-\mathrm{C}$ single-atom catalyst, which is essential for a more comprehensive understanding of how the single-atom catalyst works. To further enhance the oxygen electrochemical process of the $\mathrm{Fe}-\mathrm{N}$ active sites in ZABs, Sun's team [111] used ZIF-8 to synthesize singleatom $\mathrm{Fe}-\mathrm{N}_{x}-\mathrm{C}$ electrocatalyst by in-situ incorporating $\mathrm{Fe}^{2+}$ ions coordinated with 1,10-phenan-throline complexes (Fe-Phen) into the nanocages during the growth of ZIF-8 followed by pyrolysis (Fig. 3e). Owing to the Fe-Phen species providing both $\mathrm{Fe}^{2+}$ and organic ligands (Phen), the Fe-Phen@ZIF-8 precursor played an important role in the preparation of single-atom catalysts. After the pyrolysis at $900{ }^{\circ} \mathrm{C}$ under argon atmosphere, the precursor was converted into the isolated $\mathrm{Fe}$ single-atom on nitrogen-doped carbon frameworks, which allowed the iron atoms to be more dispersed to obtain single-atom to increase the active sites. The 
obtained $\mathrm{Fe}-\mathrm{N}_{x}-\mathrm{C}$ exhibited a higher half-wave potential of $0.91 \mathrm{~V}$ versus RHE than that of commercial Pt/C $(0.82 \mathrm{~V})$. As a cathode catalyst in the primary ZABs, it exhibited an excellent electrochemical performance at a high open-circuit voltage of $1.51 \mathrm{~V}$. In rechargeable ZABs, it showed marvelous cycling performance within $300 \mathrm{~h}$ with an initial roundtrip efficiency of 59.6\%. In addition, all-solid-state ZABs with $\mathrm{Fe}-\mathrm{N}_{x}-\mathrm{C}$ catalyst show $1.49 \mathrm{~V}$ and the cycle life was up to $120 \mathrm{~h}$.

Besides, modifying the Fe-based single-atom with appropriate MOFs, engineered morphology of the oxygen catalyst is equally conducive to optimize the ZABs performance by enhancing the mass diffusion and electron transfer between the catalyst layers, thereby increasing the utilization of active centers. As a proof-of-concept study, Hou et al. designed an overhang structure that modified an isolated monoatomic iron site by a silica-mediated MOF template method for ORR (Fig. 3f) [112]. It was found that $\mathrm{SiO}_{2}$ coated MOFs could generate outward adsorption force, causing the anisotropic thermal contraction of the MOF precursor. The dodecahedron plane collapsed during the pyrolysis process, and the fringe of ZIF-8 was maintained. At the same time, the obtained $\mathrm{N}$-doped carbon could reduce $\mathrm{Fe}^{3+}$ ions and combine with adjacent N/C atoms to form $\mathrm{Fe}-\mathrm{N}_{4}-\mathrm{C}$ sites. The as-prepared oxygen catalyst showed good performance in the ZABs, reaching a capacity of $807.5 \mathrm{mAh} \mathrm{g}^{-1}$, a high peak power density of $186.8 \mathrm{~mW} \mathrm{~cm}^{-2}$ and a decent energy density of $962.7 \mathrm{Wh} \mathrm{kg}^{-1}$, which is comparable to the $\mathrm{Pt} / \mathrm{C}$ catalyst (Fig. 3g, h). This superior activity of the MOFderived Fe SACs was contributed by the rich edge structure, effective three-phase boundaries, which enhanced the mass transport of reactants to accessible monoatomic iron sites. All the above works prove the advantage and practicability of MOFs as platform materials for synthesis effective Febased SACs as oxygen electrocatalysts for the ZABs.

\subsubsection{MOF-Derived Mn-Based SACs}

In addition to Co-based and Fe-based single-atom catalysts, many other single-atom catalysts have also attracted great attention, such as Mn-based catalysts. With the assistance of theoretical calculation, Lin et al. [113] found that the local coordination environment could adjust its intrinsic catalytic activity by changing the electronic structure of the Mn center. Furthermore, the high activity of the
pyridine-N-coordination Mn configuration was found to originate from the moderate adsorption strength of the ORR intermediate and the reasonable position of the d-band center, which promoted the ORR process. To prove such theoretical results, they successfully prepared Mn-SA electrocatalysts composed of atomically dispersed pyridine-N coordination $\mathrm{Mn}$ atoms in the carbon skeleton. The electrochemical tests showed that the Mn-based SACs had an excellent performance on ORR showing promising halfwave potential of $0.87 \mathrm{~V}$ and diffusion current-limiting performance of ZABs under alkaline conditions, which was superior to most Mn-based nanomaterial catalysts and Pt/C catalysts. More recently, well-dispersed Mn single-atoms anchored nitrogen-doped carbon with $\mathrm{Mn}-\mathrm{N}_{4}$ configuration catalyst (Mn-SAS/CN) was prepared by one-step thermal activation of $\mathrm{Mn}\left(\mathrm{CH}_{3} \mathrm{COO}\right)_{2} @ \mathrm{ZIF}-8$ precursor [114]. The operando X-ray absorption spectroscopy analysis showed that the active sites of Mn changed with the applied potential under the basic ORR condition, and $\mathrm{Mn}^{\mathrm{L}+}-\mathrm{N}_{4}$ without covering $\mathrm{OH}_{\text {ads }}$ was the catalytic center. Further DFT calculations showed that the excellent ORR performance is attributed to the easier electron transfer from $\mathrm{Mn}^{\mathrm{L}+}-\mathrm{N}_{4}$ site to the adsorbed $* \mathrm{OH}$ species. The preparation of single-atom materials is different from other materials, and the special structure of MOFs makes the probability of uniform dispersion of single-atom. Impressively, the $\mathrm{Mn}-\mathrm{N}_{4}$ oxygen electrocatalysis material demonstrated high peak power density and excellent durability when assembled in ZABs, much higher than the bulk of Fe- and Co-based SACs and the commercial $\mathrm{Pt} / \mathrm{C}$, showing great potential to replace $\mathrm{Pt}$ in practical energy devices.

\subsection{MOF-Derived Metal Cluster/Carbon Composites}

Although MOFs derived metal-free carbon materials possess better structural advantages than common carbon materials, the main problem of metal-free carbon materials is the lack of metal catalytic centers, which makes their reaction kinetics relatively slow due to the low activity of catalytic sites. Moreover, the MOFs derived metal-free materials are required to remove the metal elements within the electrocatalysts, making the synthesis process complicated and expensive. As for MOF-derived single-atom catalysts mentioned earlier, it is reported the surface area of monatomic metals increases significantly in single-atom catalyst, which 
leads to the sharp increase of surface free energy. Therefore, a large number of metal atoms are prone to agglomerate and inevitably lead to the deactivation of the catalyst during the oxygen electrolysis process [115]. Also, the limited loading of single-atom catalyst makes it far from being able to meet the practical application. Whereas MOF-derived carbon composites, doping with transition metal clusters present a feasible and promising way for the strong binding force between carbon and metal ions makes the transition metals in metal/carbon composites have higher dispersion, more active sites and faster charge transfer, thus enhancing the catalytic performance. Therefore, developing highly efficient electrolysis materials based on MOF-derived heteroatomdoped carbon matrix, incorporated with transition metals is significantly necessary for advancing the oxygen electrochemical process in ZABs [116].

\subsubsection{MOF-Derived Transition Metal Doped Carbon Composites}

Incorporation of transition metal clusters (e.g., $\mathrm{Fe}, \mathrm{Co}, \mathrm{Ni}$ ) into the carbon matrix has been demonstrated to be effective for oxygen electrocatalysis [117-119]. Owing to the diversity of MOFs, it is promising to synthesis efficient MOFderived metal/carbon composites with controlled structure by selecting proper MOF precursor and post treatment process [120]. ZIFs, as molecular sieve polyhedral nanocrystals, are a kind of widely used template to synthesis Co incorporated nitrogen-containing carbon materials [121, 122]. In the presence of ZIF-67 filled silica nanoparticles and an additional high-temperature decomposable nitrogen source, a nitrogen-rich hollow carbon cage composite material containing Co nanoparticles (Co@NHCC) was synthesized [123]. When Co@NHCC-800 was used as a gas-permeable electrode of ZABs, its open-circuit voltage was as high as $1.49 \mathrm{~V}$, and the discharge peak power density was as high as $248 \mathrm{~mW} \mathrm{~cm}{ }^{-2}$. The charge and discharge voltage gap after $12 \mathrm{~h}$ of cycling only increased by $0.1 \mathrm{~V}$. In parallel with cobalt element, Lai et al. [124] used a simple and effective $\mathrm{Cu}$ coordinated MOF strategy to prepare the novel copper-nitrogen-carbon electrocatalyst $(\mathrm{Cu}-\mathrm{N} / \mathrm{C})$ by direct pyrolysis of $\mathrm{Cu}$-doped ZIF-8 polyhedron in an inert atmosphere without a template or surfactant (Fig. 4a). As shown in Fig. 4b, 25\% Cu-N/C has higher operating durability than $30 \mathrm{wt} \% \mathrm{Pt} / \mathrm{C}$ catalyst. By supplementing the zinc anode and electrolyte, the constructed ZABs could work for a long time. After three times of so-called mechanical charging (Fig. 4c), the output voltage remained extremely stable without a significant drop, suggesting the great advantages of MOF-derived $\mathrm{Cu}-\mathrm{N} / \mathrm{C}$ in replacing precious metalbased materials in ZABs and other related energy conversion devices.

Besides, owing to their similar topological structures and unit cell parameters of ZIF-67 and ZIF-8, several works explored the potential of using these MOF hybrids as a platform to prepare transition metal doped carbon composites [125-130]. For example, the Yin group [131] put forward the synthesis of MO-Co@N-doped carbon (M means metal $\mathrm{Zn}$ or Co) by the pyrolysis of Co-contained ZIF-67 and Zncontained ZIF-8 hybrid MOFs. With the only Zn as precursors, the pyridinic $\mathrm{N}$ as an active sites for ORR formed on the surface, while, the addition of Co metal node, the OER catalytic active species $\mathrm{Co}-\mathrm{N}_{x}$ and $\mathrm{Co}^{3+} / \mathrm{Co}^{2+}$ were generated. Also, the synergy between the $\mathrm{Zn}$ and Co could promote the growth of multi-walled carbon nanotubes at high temperatures (greater than $700{ }^{\circ} \mathrm{C}$ ), which was beneficial to charge transfer. It is demonstrated that the optimized CoZnNC-700 showed excellent ZABs performance better than the $\mathrm{Pt} / \mathrm{C}$ and $\mathrm{IrO}_{2}$ hybrid batteries. Another work also explored the merits of such hybrid MOF-derived carbon nanotubes [132] by employing two-dimensional bimetallic leaf-like zeolite-based imidazolate skeletons (ZIF-L) as precursors to prepare carbon nanotubes decorated with Co nanoparticles (Co-N-CNTs). When used as dual-function oxygen electrocatalysts for the air electrode in ZABs, the primary batteries exhibited a high open-circuit potential of $1.365 \mathrm{~V}$, a current density of about $90 \mathrm{~mA} \mathrm{~cm}{ }^{-2}$, and a peak power density of about $101 \mathrm{~mW} \mathrm{~cm}^{-2}$. All these works evidenced the priority of ZIF-derived carbon nanotubes for ZABs in comparison to the commercial carbon nanotubes and $\mathrm{Pt} / \mathrm{C}$ or $\mathrm{IrO}_{2}$, suggesting the MOFs.

$\mathrm{Ni}$ nanoclusters are also frequently used as catalytic active species for oxygen electrolysis as compared to $\mathrm{Co}$, the addition of $\mathrm{Ni}$ would offer the catalysts optimized electron structure and thus enhanced electrochemical performance. By employing a multi-shelled two-dimensional NiCo-MOF@ZIF-L(Zn)@ZIF-67 composite, Co/Ni-doped porous carbon was successfully synthesized [133]. NiCo$\mathrm{NC}$ as the air electrode catalyst in the ZABs shows a large specific capacity of $792.8 \mathrm{mAh} \mathrm{g}^{-1}$, a peak power density of $243.4 \mathrm{~mW} \mathrm{~cm}^{-2}$, a small charge and discharge voltage 
(a)

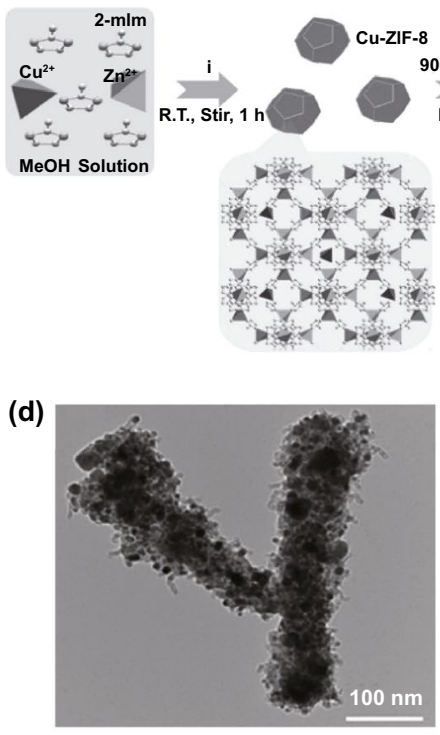

(f)

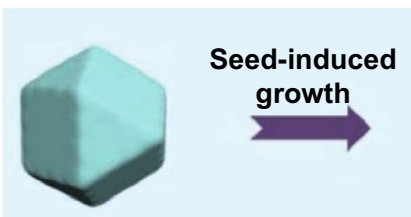

Zn-ZIF-8

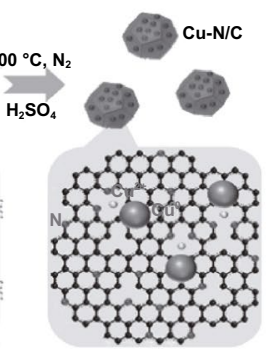

(b) 100

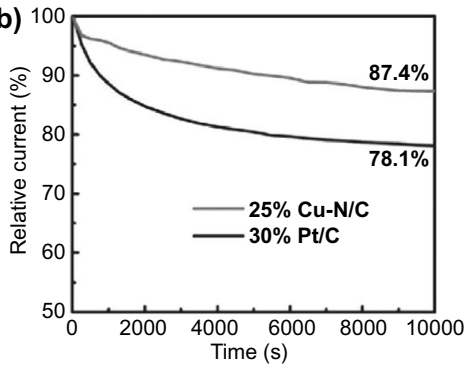

(c)

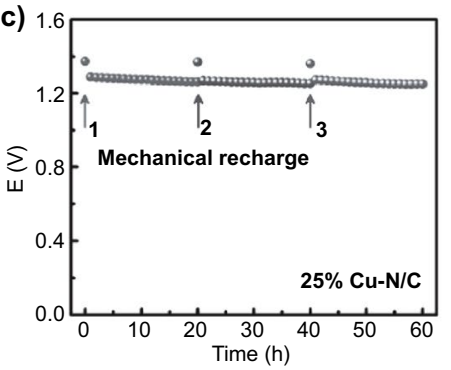

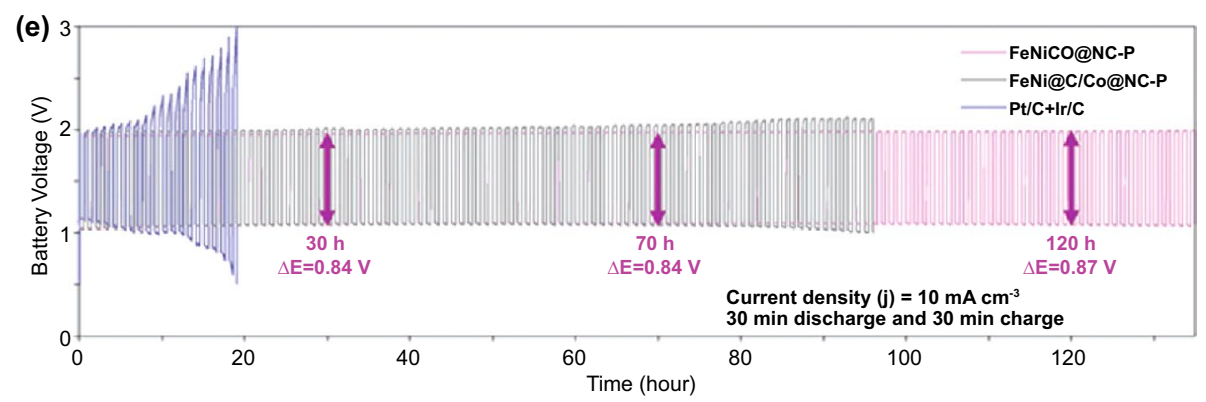

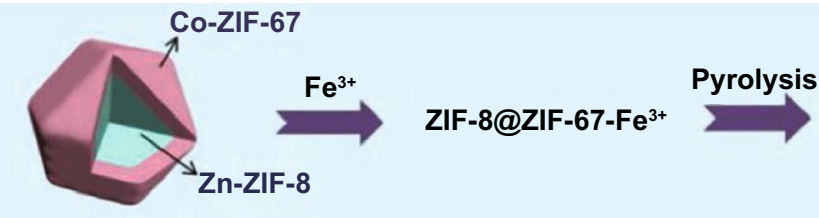

Core@shell MOFs
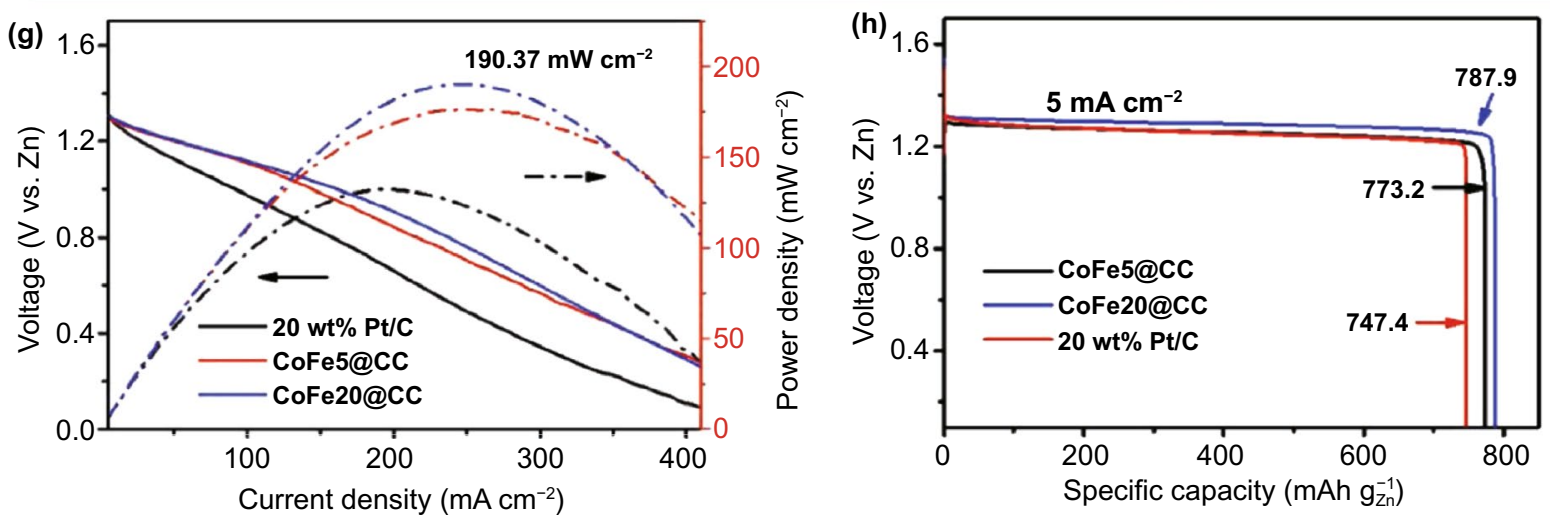

Fig. 4 a-c Synthesis process, current-time response for ORR and ZABs performance of the Cu-N/C catalyst. Copyright $\odot 2017$ Wiley-VCH Verlag GmbH \& Co. KGaA, Weinheim. d TEM image of FeNiCo@ NC-P catalyst. Copyright @ 2017 WILEY-VCH Verlag GmbH \& Co. KGaA, Weinheim. e Cycling stability for the ZABs with FeNiCo@NC-P. Copyright @ 2019 WILEY-VCH Verlag GmbH \& Co. KGaA, Weinheim. f-h Synthesis diagram, power density plots and the specific capacities of forming open carbon cages embroidered spherical structure in ZABs. Copyright @ 2019 WILEY-VCH Verlag GmbH \& Co. KGaA, Weinheim

gap of $0.84 \mathrm{~V}$ at $20 \mathrm{~mA} \mathrm{~cm}{ }^{-2}$, with good cycling stability. Thus, the design and construction of multi-shell 2D MOFs provide new opportunities for energy conversion and storage applications. Besides, trimetallic catalysts are also studied for oxygen catalysis for the potential synergetic effect [134]. Therefore, it is necessary to design dual- or multi-MOFs to achieve a good adjustment of porosity and increased intrinsic activity through the synergistic effect of multi-metal components. Chen et al. [61] proposed a new strategy for the preparation of multi-metal-based porous carbon nanorod composites by the combination of bifunctional MOFs (FeNiCo@NC-P, Fig. 4d). Compared with a single MOF-derived carbon material, $\mathrm{N}$ dopants and various metals such as $\mathrm{Fe}, \mathrm{Co}$ and $\mathrm{Ni}$ were added to the prepared catalyst. 
At the same time, the micro-/mesoporous structure was successfully prepared using two MOF precursors, and the one-dimensional carbon nanorod structure was maintained during pyrolysis at high temperatures. All these characteristics ensure that FeNiCo@NC-P had highly active ORR and OER sites, fast charge transfer, reduced interface resistance, effective oxygen and electrolyte diffusion to show excellent dual-function electrocatalytic activity. In addition, the practical application of FeNiCo@NC-P in ZABs shows a low voltage gap and long-term durability of more than $130 \mathrm{~h}$ at $10 \mathrm{~mA} \mathrm{~cm}^{-2}$ (Fig. 4e), and its performance was better than commercial precious metal benchmarks. These works not only provide a competitive dual-function oxygen electrocatalyst for ZABs but also open up new ways to design and prepare MOF-derived metal cluster/carbon composite materials with adjustable catalytic performance.

\subsubsection{MOF-Derived Alloy Doped Carbon Composites}

The formation of alloy is another effective method to improve oxygen electrocatalytic activity. The excellent catalytic activity is attributed to the synergistic effect of various metal components, which provides a variety of well-defined active sites to promote the electrocatalytic reaction [135]. Bimetallic alloys such as CoNi, FeNi or FeCo combined with carbon materials have been considered to have higher activity and stability than their single-element counterparts. At the same time, the alloy particles can improve graphitization and promote electron transfer during carbonization, while carbon carrier can prevent acid corrosion, surface oxidation and agglomeration of alloy particles. MOFs have a variety of characteristics and can be transformed into alloycarbon compounds in-situ under heat treatment, which has become an ideal precursor for designing electrocatalysts [136].

Recently, MOF-derived FeCo alloys have been extensively studied for oxygen electrocatalysis. By using amorphous ZIF-67 decorated amorphous fibers as precursors, Niu and Yang [137] firstly synthesized mesoporous nitrogen-doped graphene (MNG) compounds, then the MNG-CoFe prepared by decorating the MNG with graphene-coated CoFe alloy nanoparticles. As expected, the obtained MNG-CoFe assembled air electrodes in the ZABs showed high power density and cycle durability. In another work, the authors prepared nitrogen-doped carbon nanofibers coated with FeCo alloy nanoparticles by the pyrolysis of the CoFe-PBA@PAN precursor in argon atmosphere at $800{ }^{\circ} \mathrm{C}$ [138]. The peak power density of the ZABs based on FeCo-NCNFs-800 was as high as $74 \mathrm{~mW} \mathrm{~cm}^{-2}$, with the strong cycle stability upto 125 cycles for $42 \mathrm{~h}$. They claimed that the 1D fibrous structure, FeCo alloy nanoparticles, extensive mesoporous structure and sufficient $\mathrm{Co}-\mathrm{N}$ (pyridine-N) catalytic active sites were responsible for the high-performance ZABs. In parallel, by introducing guest iron ions into core-shell Zn@Co-MOFs precursor followed by in-situ pyrolysis, the open carbon cage self-assembled into a hydrangea-like three-dimensional superstructure connected by carbon nanotubes decorated with FeCo alloy nanoparticles (Fig. 4f) [139]. Surely, its excellent electrocatalytic performance was related to the unique superstructure, both conductive and porous channels to achieve rapid electron transfer and effective mass transfer, as well as abundant $\mathrm{Co} / \mathrm{Fe}$ catalytic sites and significant synergistic effects. As an air electrode catalyst in the ZABs, it showed perfect performance, reaching a high peak power density of $190.3 \mathrm{~mW} \mathrm{~cm}^{-2}$, an ultra-high capacity of $787.9 \mathrm{mAh} \mathrm{g}^{-1}$ and an amazing energy density of $1012 \mathrm{Wh} \mathrm{kg}^{-1}$ (Fig. 4g, h).

Although MOF-derived alloy doped carbon materials show promising oxygen electrocatalytic performance in $\mathrm{ZABs}$, design and synthesis is still in their infancy, facing different challenges. One of the big challenges is the side effects of aggregation and phase separation during the reduction process of the bimetallic phase under heat treatment. The second is the limited coordination of metal ions and organic ligands, which limits the choice of metal-ligand combinations [140, 141]. Therefore, more emerging strategies need to be developed to further discover MOF-derived alloy-carbon catalysts with unique catalytic properties. Meanwhile, the suitable metal precursors with the required proportion and composition can be selected by the prediction of the phase diagram and enthalpy of formation and can coordinate with MOF ligands through different reactions such as gas-phase, liquid-phase and solid-phase [135].

\subsection{MOF-Derived Metal Compound/Carbon Composites}

For the past few years, transition metal compounds have long been studied as oxygen electrocatalysts, but the inherent low conductivity and poor dispersion of nanoparticles seriously hinder their electrocatalytic activity. Consequently, 
transition metal compound combined with carbon materials, MOF-derived carbon materials particularly, can effectively overcome the above concerns [142-144]. By far, various MOF-derived metal compound/carbon composites have been reported for oxygen electrocatalysis, which mainly includes metal oxides, metal sulfides/carbides/phosphides and other metal compounds composite carbon materials.

\subsubsection{Metal Oxides}

Transition metal oxides are the most widely studied compounds for oxygen electrolysis. On the one hand, they are easy to prepare in oxidizing or alkaline environments, on the other hand, they are very stable during the oxygen electrocatalytic process due to the oxidized surface composition. When MOFs employed as precursors, oxygen-rich vacant metal oxides can be obtained, which would offer much higher ORR/OER activity than ordinary materials. Among various MOFs, Co containing MOFs are mostly studied. These MOF-derived cobalt oxide/carbon composite material can help to improve the conductivity and dispersibility of the cobalt oxide, thereby exposing the intrinsic active sites in the catalyst [145]. Compared with pure $\mathrm{Co}_{3} \mathrm{O}_{4}$, the MOF-derived hybrid carbon material based on $\mathrm{Co}_{3} \mathrm{O}_{4}$ doped with nitrogen, sulfur, and phosphorus showed better electrochemical activity [146-152]. For instance, Ren et al. [153] prepared a porous nanowire array composed of $\mathrm{Co}_{3} \mathrm{O}_{4}$ nanoparticles and carbon species by carbonizing ZIF-67 directly grown on nickel foam. The resulting hybrid material was used as an air catalyst for ZABs, which exhibited a large peak power density of $118 \mathrm{~mW} \mathrm{~cm}^{-2}$ and promising operating stability.

Designing unique nanostructures also play a significant role in the improvement of oxygen catalytic activity. By using a well-designed MOFs precursor, a N-doped carbon nanowall array embedded with irregular hollow $\mathrm{Co}_{3} \mathrm{O}_{4}$ nanospheres electrocatalyst $\left(\mathrm{NC}-\mathrm{Co}_{3} \mathrm{O}_{4}\right)$ was designed [154]. The authors explained that the surface of the metal nanoparticles covered with a layer of graphite onion during the carbonization process (Fig. 5a). Then the carbon onion-coated Co nanoparticles inhibited the Kirkendall effect at the nanoscale, promoted the formation of irregular hollow $\mathrm{Co}_{3} \mathrm{O}_{4}$ nanospheres, and offered them pleasurable catalytic properties for OER and ORR.
Furthermore, the integrated $\mathrm{NC}-\mathrm{Co}_{3} \mathrm{O}_{4} / \mathrm{CC}$ was directly used as an additive-free air cathode for flexible allsolid ZABs, showing a large capacity of $387.2 \mathrm{mAh} \mathrm{g}^{-1}$ (Fig. 5b), exceptional cycle stability and mechanical flexibility, which was significantly better than Pt-based and infrared ZABs. In another work, by a facile carbonization-oxidation method, the 3D-on-2D MOF precursor on carbon cloth (ZIF-L-D/CC) was converted to nitrogen-doped carbon and $\mathrm{Co}_{3} \mathrm{O}_{4}$ nanoparticles (ZIF-L-D$\mathrm{Co}_{3} \mathrm{O}_{4} / \mathrm{CC}$ ) [155]. This unique layered MOF on MOF (3D-on-2D) structure undoubtedly promoted the reaction kinetics and proton transport. Meanwhile, the tight protection of $\mathrm{Co}_{3} \mathrm{O}_{4}$ nanoparticles by $\mathrm{N}$-doped carbon also provided prominent electrochemical activity and stability. In particular, when the catalyst was applied to air cathode inflexible all-solid-state ZABs, it exhibited high open-circuit potential $(1.461 \mathrm{~V})$, capacity $\left(815 \mathrm{mAh} \mathrm{g}^{-1}\right.$ at $1 \mathrm{~mA} \mathrm{~cm}^{-2}$ ), energy density (1010 $\left.\mathrm{Wh} \mathrm{kg}^{-1}\right)$, exceptional cycling stability as well as outstanding mechanical flexibility. This work could push the enormous development of new-type flexible energy conversion and storage devices.

In addition to cobalt oxide, manganese oxide is another promising oxygen electrocatalytic materials for its variable geometry. To resolve the low conductivity of single manganese oxide, Chen et al. [156] selected ZIF-67 supported ultra-thin $\mathrm{MnO}_{2}$ hollow nanowire as a precursor, $\mathrm{MnO} @$ Co-N/C nanomaterials with controllable diameter were obtained after pyrolysis. The synergistic effect between the $\mathrm{MnO}$ and porous $\mathrm{Co}-\mathrm{N} / \mathrm{C}$ provide excellent catalytic activity and performance of the $\mathrm{ZABs}$, even better than $\mathrm{Pt} / \mathrm{C}$ and $\mathrm{RuO}_{2}$ mixed catalysts. To further investigate such MOFderived $\mathrm{MnO} / \mathrm{Co}$ for oxygen electrolysis, a heterogeneous $\mathrm{MnO} / \mathrm{Co}$ interface in a porous graphite carbon polyhedron ( $\mathrm{MnO} / \mathrm{Co} / \mathrm{PGC})$ was prepared by using a bimetallic metal-organic framework as a precursor [157]. In-situ generated Co nanocrystals could not only form a highly conductive heterointerface, overcome the disadvantage of poor OER activity, but also promote the formation of graphitic carbon. Therefore, MnO/Co/PGC shows excellent activity and stability for both OER and ORR. Importantly, domestic ZABs exhibited outstanding performance, including peak power density of $172 \mathrm{~mW} \mathrm{~cm} \mathrm{~cm}^{-2}$, specific capacity of 872 $\mathrm{mAh} \mathrm{g}^{-1}$, as well as excellent cycle stability, better than commercial Pt/C mixed catalyst with $\mathrm{RuO}_{2}$. Different from 
(a)
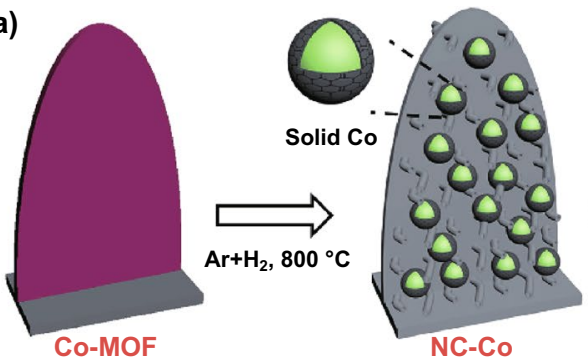

(c)

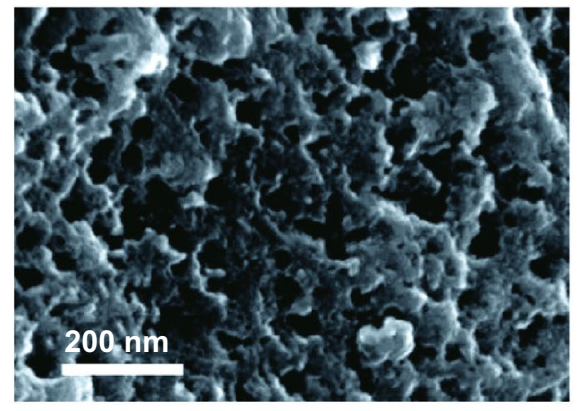

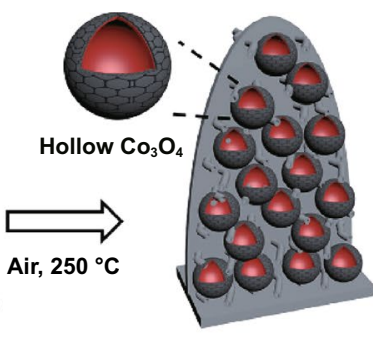

$\mathrm{NC}-\mathrm{CO}_{3} \mathrm{O}_{4}$

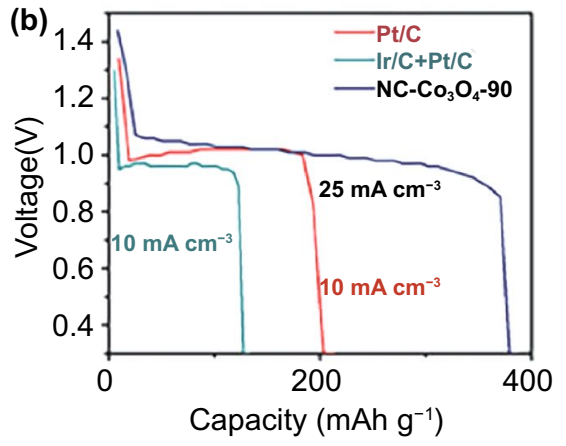

(d)

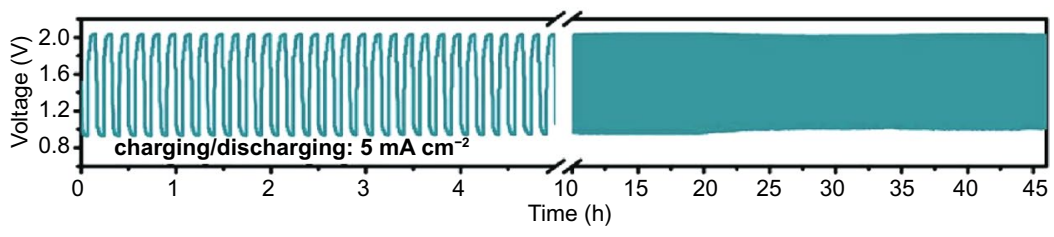

(e)
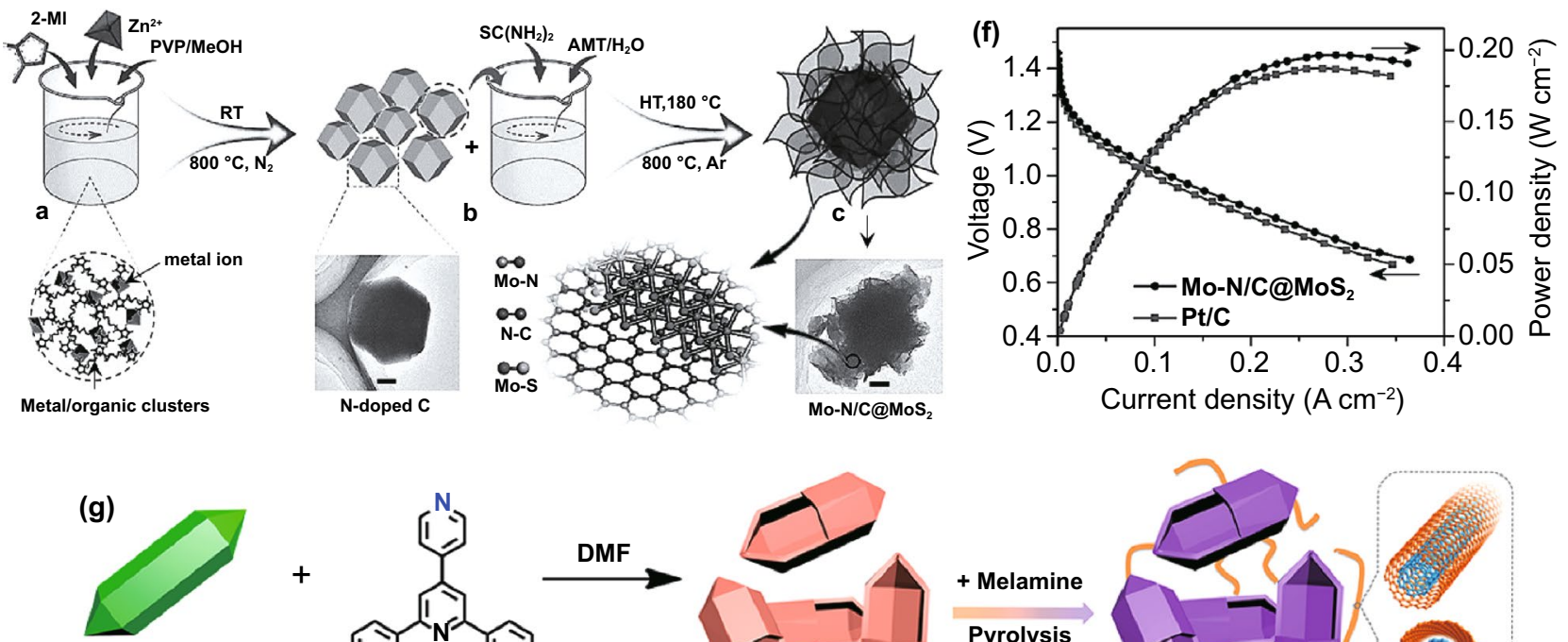

FeNi-MIL-88B

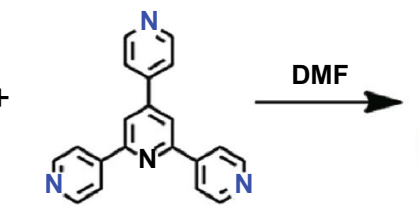

tpy
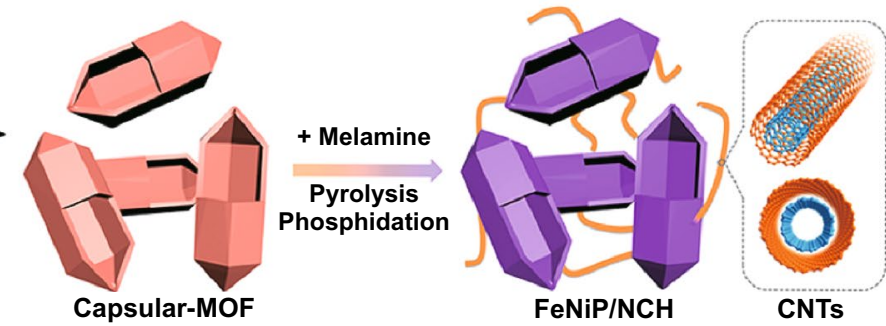

Fig. 5 a Fabrication process and $\mathbf{b}$ voltage-capacity curves (solid-state $\mathrm{Zn}$-air batteries) for hierarchical $\mathrm{NC}^{-\mathrm{Co}_{3} \mathrm{O}_{4}}$ arrays on flexible carbon cloth. Copyright (C) 2017 WILEY-VCH Verlag GmbH \& Co. KGaA, Weinheim. c SEM image and d long-term charging-discharging cycling tests of $\mathrm{Co}_{9} \mathrm{~S}_{8} @$ TDC-900 catalyst. Copyright (c) 2019 The Royal Society of Chemistry. e Schematics for the synthesis and $\mathbf{f}$ V-I polarization and power density curves of Mo-N/C@ $\mathrm{MoS}_{2}$ catalyst. Copyright @ 2017 WILEY-VCH Verlag GmbH \& Co. KGaA, Weinheim. g Preparation process diagram of FeNiP/NCH catalyst. Copyright (c) 2019 American Chemical Society

the simple mixture of $\mathrm{MnO}_{2}$ with $\mathrm{MOF}$ to prepare metal oxides/carbon composite, this work emphasizes the synergy of heterogeneous interfaces in oxygen electrocatalysis, thereby providing a promising way to develop advanced zinc-air cathode materials.

\subsubsection{Metal Sulfides/Phosphides/Carbides}

MOF-derived carbon-based transition metal sulfides/ phosphides/carbides composites are also hotspots in ZABs [158]. For instance, $\mathrm{Co} / \mathrm{Co}_{x} \mathrm{~S}_{y} @ \mathrm{~S}, \mathrm{~N}$-doped carbon 
fibers were prepared by Co-MOFs fibers under hydrothermal conditions followed with pyrolysis at $800{ }^{\circ} \mathrm{C}$ [159]. The catalyst showed excellent bifunctional ORR and OER activity, as well as high power density and favorable durability in rechargeable ZABs. To further investigate such composite on the oxygen electrolysis, N, O and S-doped carbon matrix embedded with $\mathrm{Co}_{9} \mathrm{~S}_{8}$ nanoparticles $\left(\mathrm{Co}_{9} \mathrm{~S}_{8} @ \mathrm{TDC}\right.$, Fig. 5c) was produced by direct carbonization of a novel Co-MOF constructed by SPDP (4,4'-(sulfonylbis(4,1-phenylene))dipyridine) and $\mathrm{H}_{2} \mathrm{BDC}$ (1,4-benzene dicarboxylic acid) ligands [160]. The inherent activity of $\mathrm{Co}_{9} \mathrm{~S}_{8}$ nanoparticles and the heteroatomdoped carbon shell promote the catalytic performance of OER and ORR. The $\mathrm{Co}_{9} \mathrm{~S}_{8} @$ TDC-900 was used as an air cathode catalyst layer in rechargeable ZABs, which provided considerable open-circuit voltage of $1.50 \mathrm{~V}$ and long-term charge-discharge stability (Fig. 5d). In addition to the cobalt sulfide, the study on molybdenum sulfide is also quite remarkable toward ORR electrocatalysis. Considering the outstanding flexibility, ultra-high surface area, layered pore structure and high catalytic activity of MOFs and metal dihydrogen disulfide, Mu's team reported a highly efficient electrocatalyst based on a vertically aligned $\mathrm{MoS}_{2}$ nanosheet hierarchically interconnected Mo-N/C frame produced by carbonization of ZIF-8 resulting in the formation of an interface Mo-N coupling center (Fig. 5e) [161]. Surprisingly, when used as a cathode electrocatalyst in ZABs, it showed a high power density of about $196.4 \mathrm{~mW} \mathrm{~cm}^{-2}$ (Fig. 5f), and the voltammetry efficiency at $5 \mathrm{~mA} \mathrm{~cm}^{-2}$ was about $63 \%$. Even after $48 \mathrm{~h}$ at $25 \mathrm{~mA} \mathrm{~cm}^{-2}$, it still had excellent cycle stability. Such perfect electrocatalytic performance was attributed to the synergistic effect of unique chemical composition, unique three-phase active site and layered pore frame for rapid mass transfer.

Owing to the similar catalytic properties of phosphides with sulfides, there are also a few studies on MOF-derived metal phosphides/carbon composites [162, 163]. In comparison to transition metal sulfides-based oxygen electrocatalysts, phosphides display enhanced catalytic activity but limited stability, while MOFs just provide a potential solution for such issue. For example, based on the pyrolysis of ZIF67 and dicyandiamide, Hao et al. [164] synthesized carbon polyhedral penetrating bamboo-like carbon nanotubes by a three-step chemical method. This polyhedron provided large interfacial areas for catalytic reactions. The compositions of $\mathrm{Co}, \mathrm{CoP}$ and hairy $\mathrm{N}$-doped carbon in the catalyst made it possess higher catalytic activity. As an air cathode in the rechargeable ZABs, it still had high round-trip efficiency, low overpotential and stable voltage platform after 100 cycles. In another important work, the authors used MOF enveloped protein and melamine as starting materials, then a N-doped envelope carbon-based framework was prepared by the pyrolysis-phosphine reaction with the iron-nickel phosphide nanoparticles fixed to the envelope connected by a large number of carbon nanotubes on carbon (Fig. $5 \mathrm{~g}$ ) [165]. The synergistic effect between the carbon skeleton and the highly surface-exposed phosphide sites made the material exhibit highly efficient multi-functional electrocatalysis in HER, OER and ORR. It was a qualified component for rechargeable ZABs with the peak value power density was $250 \mathrm{~mW} \mathrm{~cm}^{-2}$, and perfect stability was up to $500 \mathrm{~h}$.

Apart from the transition metal sulfides/phosphides, MOF-derived carbide/carbon composites also show good electrocatalytic properties [166]. By assembling ZIF-8 nanoparticles (NPs) within the polyacrylonitrile nanofibers with an electrospinning method, Liu et al. [167] reported $\mathrm{Fe}_{3} \mathrm{C}$ NPs embedded in $\mathrm{Fe}-\mathrm{N}$-doped porous carbon nanofibers as a bifunctional oxygen electrocatalyst applied in ZABs. Similarly, by using ZIF-8 as a precursor and uniformly distributed ultrafine $\alpha-\mathrm{MoC}$ nanoparticles as a model electrocatalyst, a nitrogen-doped layered porous carbon material $(\alpha-\mathrm{MoC} /$ NHPC) was prepared [168]. Theoretical studies have shown that $\alpha-\mathrm{MoC}$ on NHPC could effectively reduce the energy barrier for proton generation during the hydrolysis process, and ultimately promote the proton coupling ORR dynamics. The $\alpha-\mathrm{MoC} / \mathrm{NHPC}$ catalyst synthesized with the assistance of $\mathrm{NaCl}$ had the advantages of ultrafine nanoparticles and MOF-derived layered porous carbon structure, it exhibited excellent ORR performance with a half-wave potential as high as $0.88 \mathrm{~V}$. As the air electrode of the ZABs, its peak

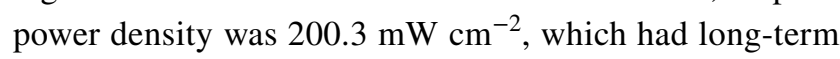
stability.

\subsubsection{Other Compounds}

$\mathrm{N}$-doping and nitrides have been proved to be active species for oxygen electrolysis by promoting the charge transfer in the catalytic process [169]. Because the pyrolysis of MOFs may lead to the loss of nitrogen content, it is feasible to enrich the amount of nitrogen in MOF derivatives by 
nitriding [170]. For instance, Guan et al.[171] demonstrated the design of $\mathrm{NC}$ embedded with $\mathrm{Co} / \mathrm{CoN}_{\mathrm{x}}$ nanoparticles $\left(\mathrm{NC}-\mathrm{Co} / \mathrm{CoN}_{x}\right.$ ) by carbonizing Co-ZIF-L followed with nitriding process (Fig. 6a), which provided abundant active sites, high-density interfaces and short ion diffusion paths. This highly integrated electrode combines the advantages of each component, showing high electrochemical performance and strong mechanical stability. A MOF-derived metal nitrides/N-doped carbon composites $\left(\mathrm{Fe}-\mathrm{Co}_{4} \mathrm{~N} @ \mathrm{~N}-\mathrm{C}\right)$ with richly accessible pyridine-N-M active sites were developed by ammonization [172]. Owing to the strong coordination between metal center and pyridinic nitrogen, Fe doping in Co-MOF promoted the formation of a large number of pyridine-N-M active sites for ORR (Fig. 6b). Therefore, when used as the air cathode in liquid $\mathrm{Zn}$-air batteries, the electrocatalyst achieved a high specific capacity of $806 \mathrm{mAh} \mathrm{g}^{-1}$ at $5 \mathrm{~mA} \mathrm{~cm}^{-2}$ (Fig. 6c) and excellent cycle stability.
Furthermore, MOF-derived metal selenides/carbon composites have also attracted enormous attention toward oxygen electrocatalysis. By direct selenization of ZIF-67, the ultrafine $\mathrm{Co}_{0.85}$ Se nanocrystals coupled with $\mathrm{N}$-doped carbon was developed [173]. In-situ carbonization of ZIF-67 could ensure a smaller size of $\mathrm{Co}_{0.85}$ Se nanocrystal to increase the active sites to obtain a higher electrocatalytic activity. Also, the $\mathrm{Co}_{0.85} \mathrm{Se} @ \mathrm{NC}$ catalyst inherited the unique porous structure of the ZIF-67 template, which could increase its surface area in contact with electrolyte, thus effectively promoting the transfer of electrolyte and the rapid diffusion of gas products for better performance. The catalyst assembled in ZABs played a very low charge-discharge voltage gap of $0.80 \mathrm{~V}$ and a life span of 180 cycles at $10 \mathrm{~mA} \mathrm{~cm}^{-2}$. Because the electron transfer of metal compound in the catalytic process can affect the intrinsic conductivity of the catalyst. Therefore, with the assistance of MOFs, both phosphorous (a)

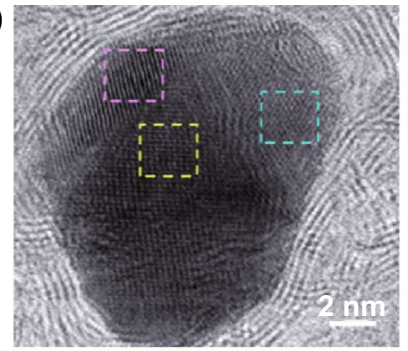

(b)

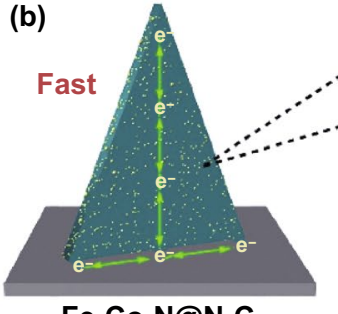

$\mathrm{Fe}-\mathrm{Co}_{4} \mathrm{~N} @ \mathrm{~N}-\mathrm{C}$
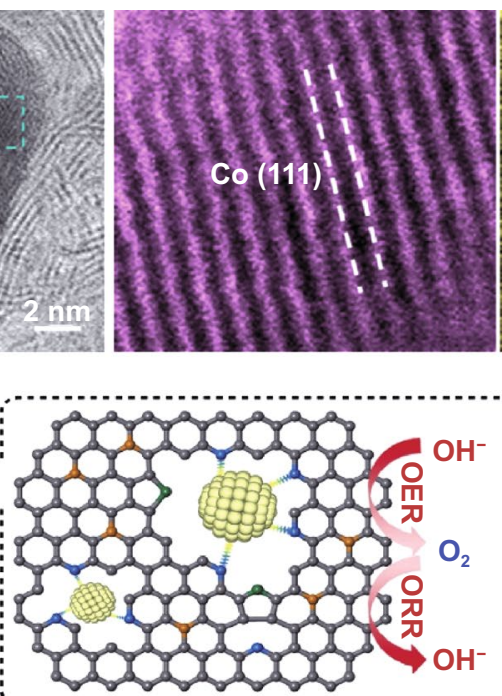
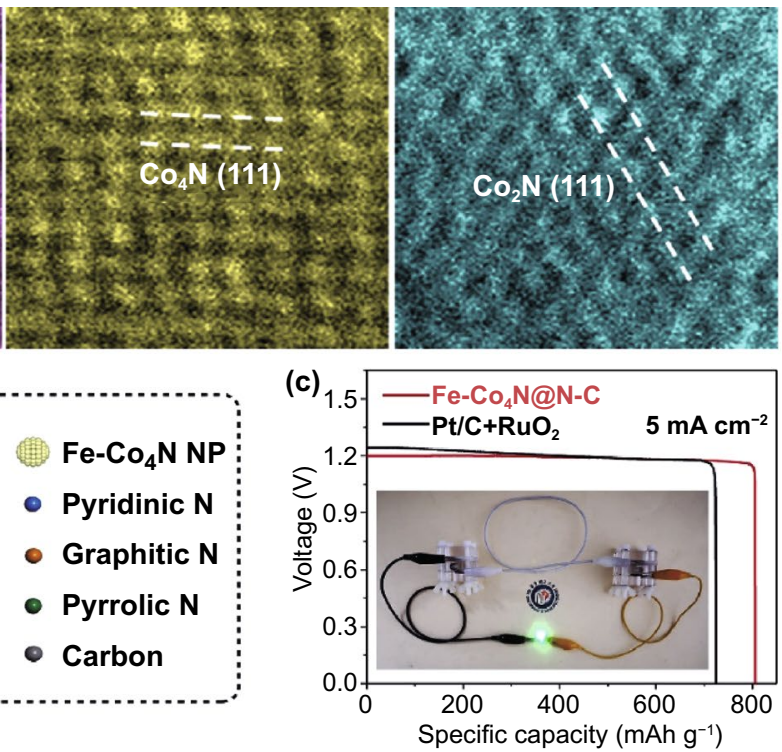

$\mathrm{Fe}-\mathrm{Co}_{4} \mathrm{~N} N \mathrm{NP}$

- Pyridinic N

- Graphitic N

- Pyrrolic N

- Carbon (d)

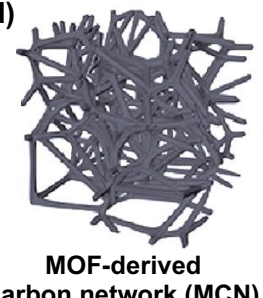

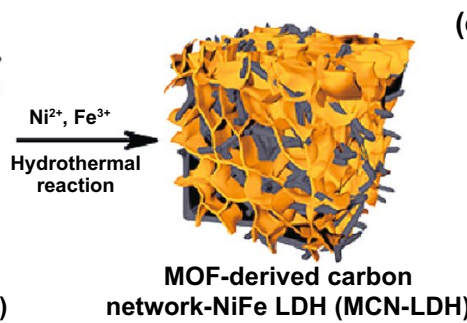

(e) 4 - Pt/C-RuO

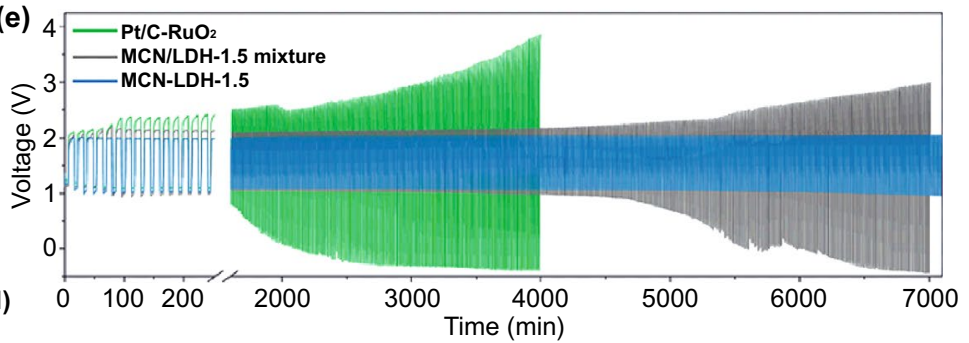

Fig. 6 a High-magnification STEM ABF image of $\mathrm{NC}-\mathrm{Co} / \mathrm{CoN}_{x}$ catalyst and the lattice fringe from different parts of it. Copyright $\odot 2018 \mathrm{Else}-$ vier B.V. b Schematic synthesis process and $\mathbf{c}$ the specific discharging capacities curves of the $\mathrm{Fe}-\mathrm{Co}_{4} \mathrm{~N} @ \mathrm{~N}-\mathrm{C}$ for air electrode. Copyright $\odot$ 2019 Elsevier B.V. d Preparation diagram and e continuous discharge/recharge voltage records of air cathodes in zinc-air catalyzed by of MOFderived carbon network-NiFe-LDH catalyst. Copyright @ 2019 American Chemical Society 
and selenium were introduced during pyrolysis to form a complex catalyst $\mathrm{CoFe}-\mathrm{P}-\mathrm{Se} / \mathrm{NC}$ [174]. A low voltage gap of $0.719 \mathrm{~V}$ at $200 \mathrm{~mA} \mathrm{~cm}^{-2}$, the high power density of 104 $\mathrm{mW} \mathrm{cm}{ }^{-2}$, and the high energy density of $805 \mathrm{Wh} \mathrm{kg}^{-1}$ were achieved when used in a rechargeable battery.

As a typical two-dimensional material, layered double hydroxides (LDHs) have long been studied for oxygen electrochemical reactions, especially the OER process, but the applications of MOF-derived hydroxides in ZABs are inadequate [175]. Recently, Qian and his colleagues explored the performance of the NiFe layered double hydroxides for ZABs [176]. They prepared the OER active NiFe-LDH composites supported on ORR active MOF-derived carbon network (MCN) as Janus-ORR/OER electrocatalyst for the rechargeable ZABs. Through simple hydrothermal reaction, LDH nanoplates could be easily fixed on MCN (Fig. 6d). The interaction between MCN and LDH enhanced the OER activity, but ORR activity remained positive. When MCN$\mathrm{LDH}$ was further installed in the rechargeable ZABs, it could operate continuously for more than $100 \mathrm{~h}$ at $10 \mathrm{~mA} \mathrm{~cm} \mathrm{~cm}^{-2}$ without significant performance loss (Fig. 6e). The life of the batteries was tripled as compared to the standard precious metal catalyst $\mathrm{Pt} / \mathrm{C}-\mathrm{RuO}_{2}$ assembled batteries. Therefore, the MOF-derived hydroxides cases show great potential in the development of the ZABs and other related electrochemical energy storage systems [177].

\section{Conclusion and Outlook}

With the increasing demand for energy conversion devices in modern society, air electrode is of immense significance for ZABs. For the improved performance and durability of ZABs, the exploration of efficient oxygen electrocatalysts is urgent and meaningful. MOFs featured with diverse inorganic metal nodes and organic ligands provide an abundant platform for the design of low cost and highly efficient oxygen electrocatalysts for the air cathode in ZABs. In this review, with a brief introduction of the fundamentals on oxygen electrolysis in ZABs, the recent advances on MOFderived non-noble metal-oxygen electrocatalysts are successively reviewed for ORR and OER from the category of metal-free carbon materials, single-atom catalysts, metal/ carbon composites and metal compound/carbon composites (Table 1). In particular, the structure-performance relationship of these MOF-derived non-noble metal oxygen electrocatalysts for zinc-air batteries (Fig. 7a) in term of their oxygen electrocatalytic activity and specific capacity. Despite great achievements have been made in the field of MOF-derived oxygen materials for zinc-air batteries, they still face a host of numerous challenges.

Here, we put forward several criteria for the commercialization of ZABs in future from the design of catalysts, electrode and batteries by considering the performance, safety and cost (Fig. 7b). Design and construction of efficient electrode materials are always of uppermost priority, the structural diversity of MOFs makes the materials system very complex, selection of proper MOF platform is highly critical for design MOF-derived oxygen electrocatalytic materials. Using high throughput technology, one can calculate and predict the possibility of the different MOF compositions and structures on the performance of final products, and thus accelerating the manufacturing speed of MOF-derived air electrode materials [178, 179]. Besides, the direct applications of MOFs electrocatalysts in ZABs are rarely studied, the ORR catalytic performance of pure MOFs has not reached the point of direct applications, with the aid of high throughput screening, the fast selection of active metal and organic ligands becomes impossible and accelerated for the design of catalytic active MOFs for ORR.

At present, the accurate structure elucidation of as-prepared MOFs derivatives is particularly useful for studying their structure-activity relationship during oxygen electrocatalysis in ZABs. Currently, growing individuals choose insitu testing technology to explain the process and mechanism of OER, ORR and other electrocatalysis [180-182]. By the combination of electrode and batteries test with advanced in-situ synchrotron radiation technology, it would appreciate to fully characterize the structural evolution of the catalysts during the electrochemical reactions and batteries service. Over the past decade, intense research activities have been made for the oxygen electrocatalysts and air cathode, and a shift of research attention from air cathodes to $\mathrm{Zn}$ anodes would also advance the current ZABs.

In addition to the aforementioned challenges, the commercialization of current ZABs requires further balance in performance, safety and cost. The improvement of performance, such as power density, energy density and cyclability, could be achieved by optimizing the electrode materials, batteries configuration, electrolytes and operation conditions. For example, developing MOFs derivatives with enhanced graphitization and open-framework or 2D intercalation MOF 


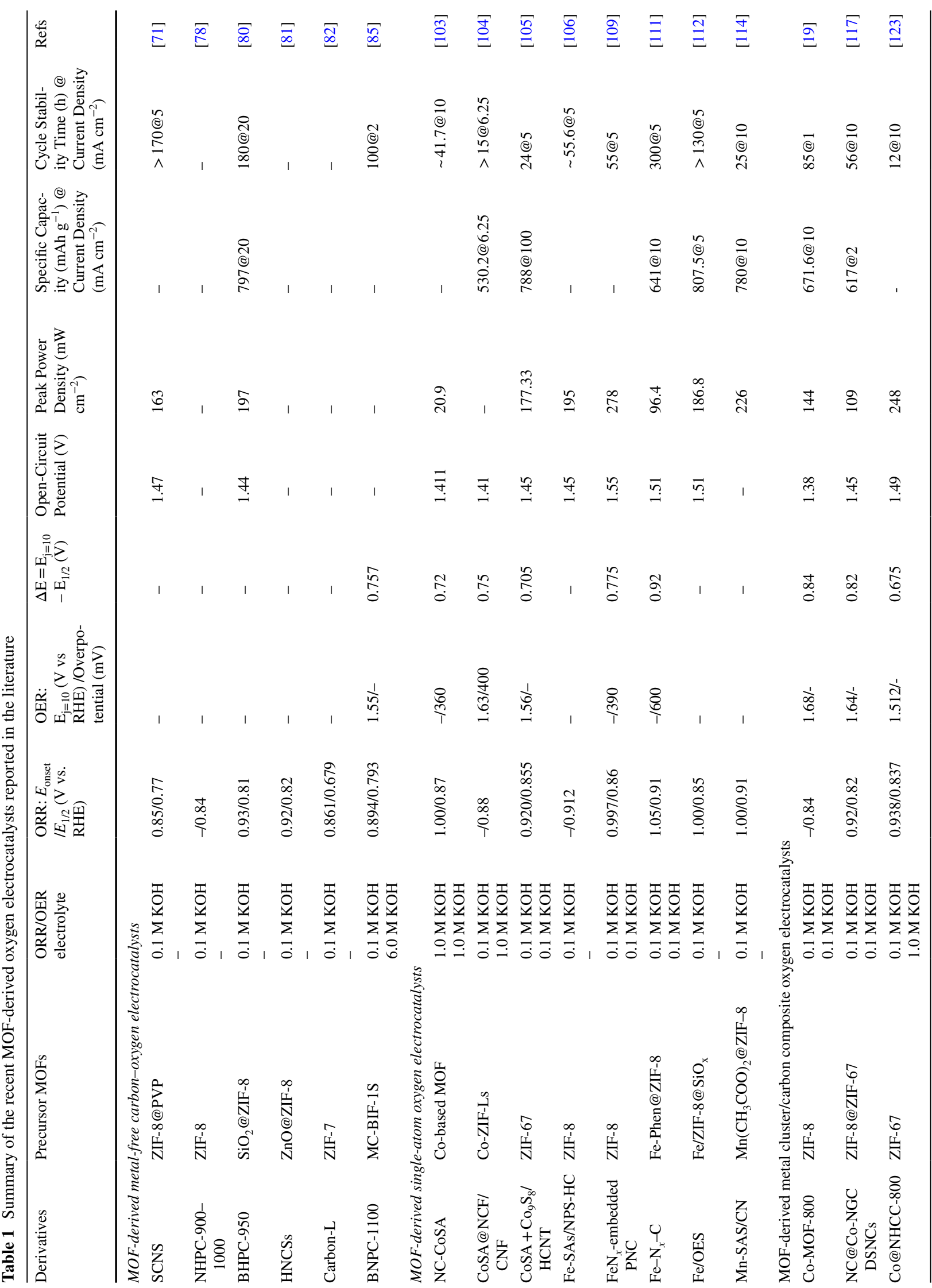




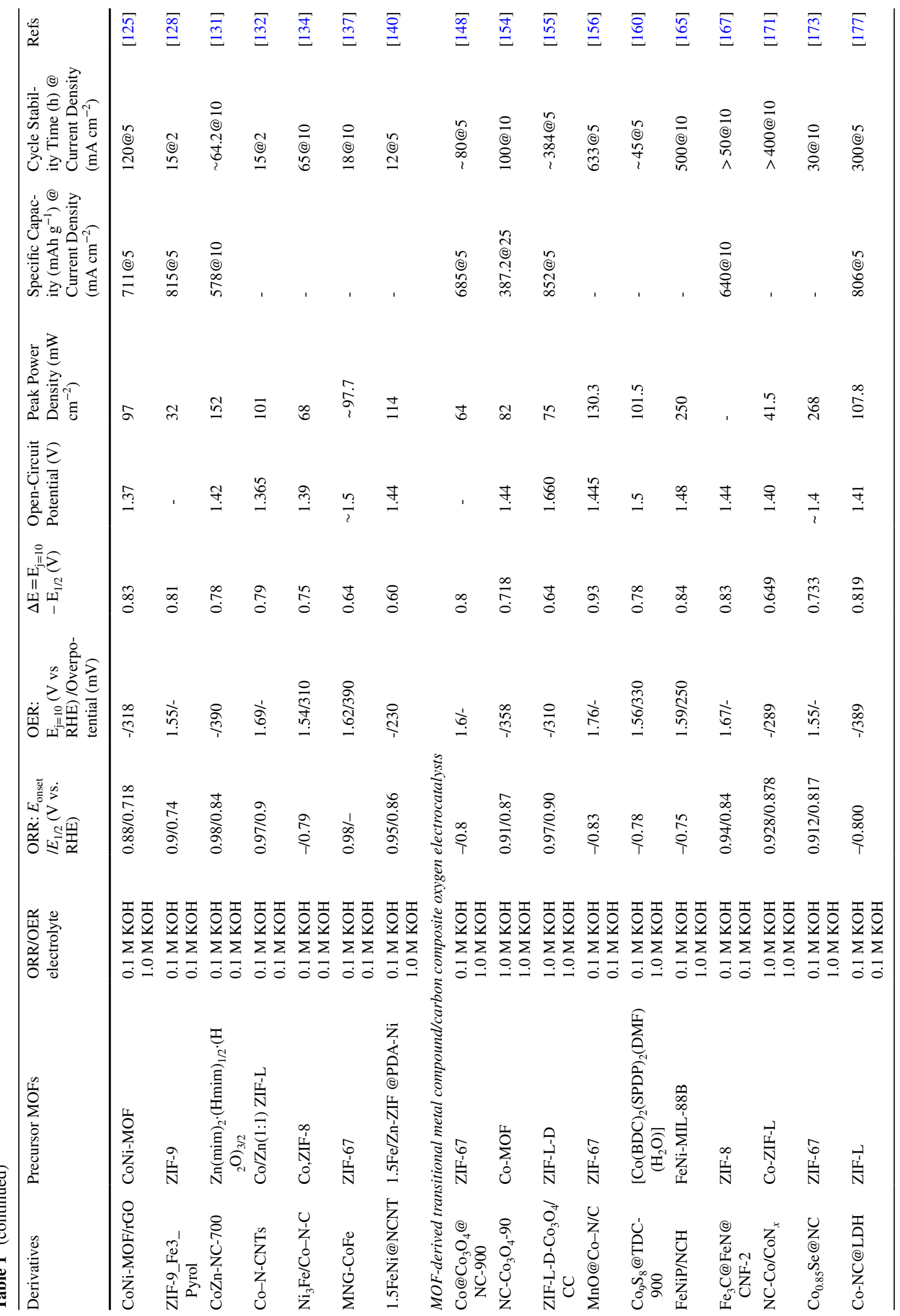




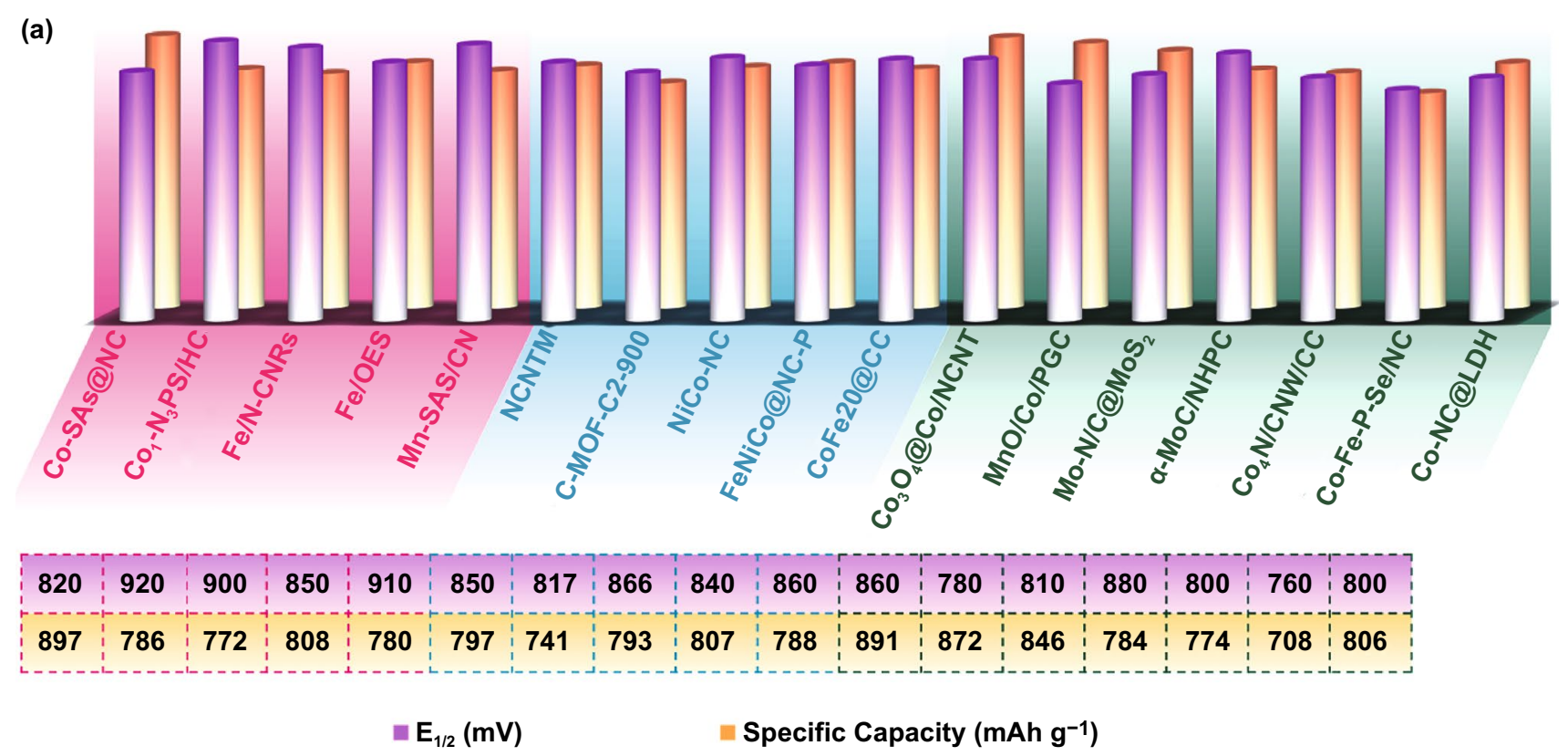

(b)

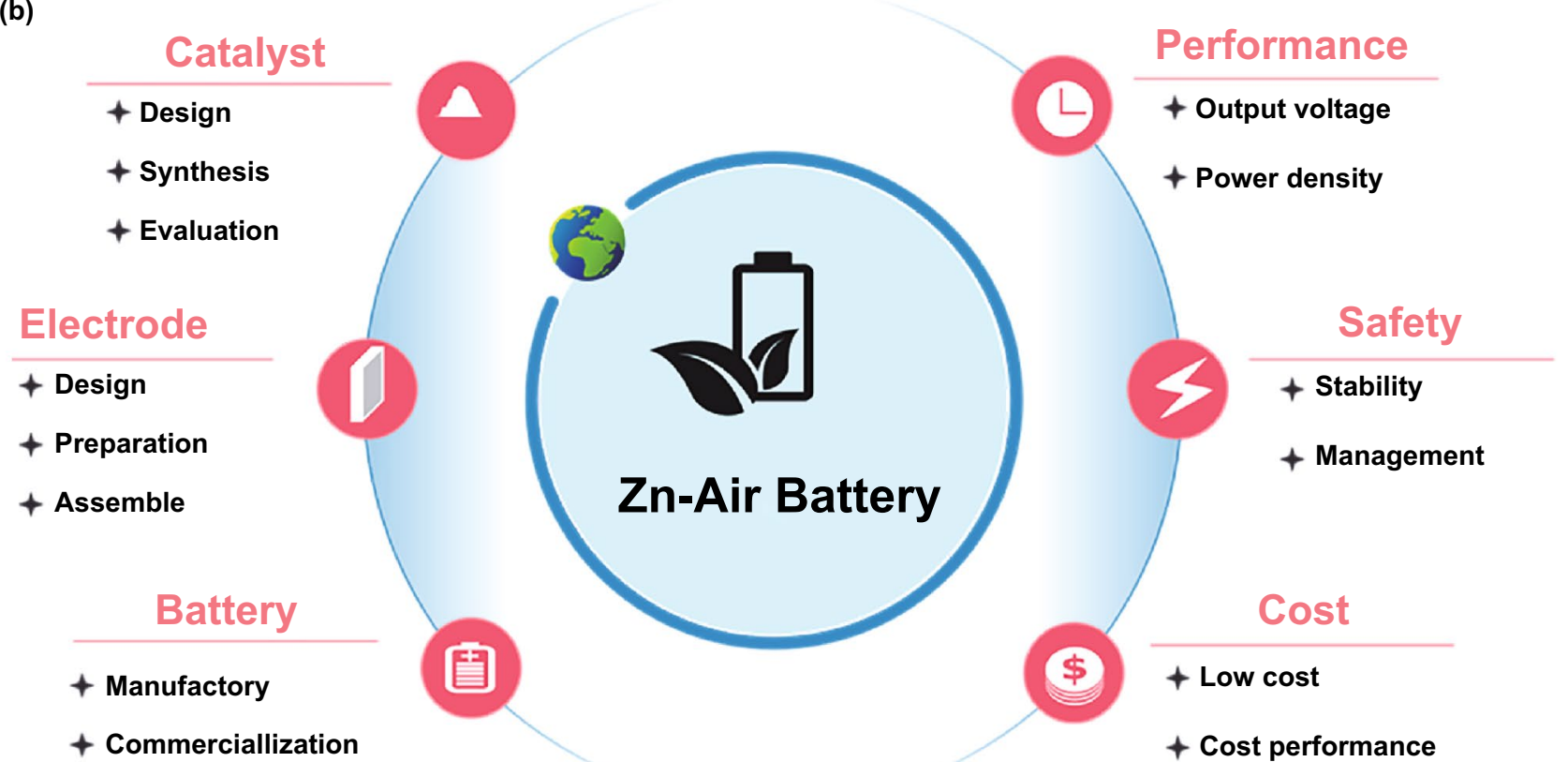

Fig. 7 a Summary of the half-wave potential $\left(E_{1 / 2}\right)$ and specific capacity for advanced MOF-derived oxygen electrocatalysts for ZABs. b Proposed aspects for the future development of ZABs

electrode with tailored architecture; improve the utilization of electrolyte and prevent leakage; widen the output voltage by controlling the overpotential. Apart from high performance, safety is always the key aspect of any future battery technology in our daily life, especially for ZABs in comparison to current Li-ion batteries. Also, low cost, determining the large-scale application of ZABs, is crucial, which can be realized by using earth-abundant resources, simple manufacturing processes and facile control systems. Therefore, achieving the balance of cost and performance is the main task for the fast-growing research on ZABs in future. 
While commercialization progress of ZABs requires more and more research work, it is believed that the progress in electrode materials innovations will boost the performance of ZABs in the coming years. At the same time, the structure of ZABs, the electrolyte and other aspects are all need to be improved so that the performance of ZABs could be further advanced in future. By considering the balance of high performance, safety and low cost, future developments in this area will surely advance the large-scale application of high-performance ZABs.

Acknowledgements This work is supported by the National Natural Science Foundation of China (22075092) and the Program for HUST Academic Frontier Youth Team (2018QYTD15).

Open Access This article is licensed under a Creative Commons Attribution 4.0 International License, which permits use, sharing, adaptation, distribution and reproduction in any medium or format, as long as you give appropriate credit to the original author(s) and the source, provide a link to the Creative Commons licence, and indicate if changes were made. The images or other third party material in this article are included in the article's Creative Commons licence, unless indicated otherwise in a credit line to the material. If material is not included in the article's Creative Commons licence and your intended use is not permitted by statutory regulation or exceeds the permitted use, you will need to obtain permission directly from the copyright holder. To view a copy of this licence, visit http://creativecommons.org/licenses/by/4.0/.

\section{References}

1. A.M. Omer, Energy, environment and sustainable development. Renew. Sust. Energy Rev. 12, 2265-2300 (2008). https://doi.org/10.1016/j.rser.2007.05.001

2. H.-X. Zhong, Q. Zhang, J. Wang, X.-B. Zhang, X.-L. Wei et al., Engineering ultrathin $\mathrm{C}_{3} \mathrm{~N}_{4}$ quantum dots on graphene as a metal-free water reduction electrocatalyst. ACS Catal. 8, 3965-3970 (2018). https://doi.org/10.1021/acscatal.8b00467

3. Y. Wang, M.M. Shi, D. Bao, F.L. Meng, Q. Zhang et al., Generating defect-rich bismuth for enhancing the rate of nitrogen electroreduction to ammonia. Angew. Chem. Int. Ed. 58, 9464-9469 (2019). https://doi.org/10.1002/anie. 201903969

4. Q. Zhang, H. Zhong, F. Meng, D. Bao, X. Zhang et al., Threedimensional interconnected $\mathrm{Ni}(\mathrm{Fe}) \mathrm{O}_{\mathrm{x}} \mathrm{H}_{\mathrm{y}}$ nanosheets on stainless steel mesh as a robust integrated oxygen evolution electrode. Nano Res. 11, 1294-1300 (2018). https://doi.org/10. 1007/s12274-017-1743-8

5. L. Zhao, Z. Liu, D. Chen, F. Liu, Z. Yang et al., Laser synthesis and microfabrication of micro/nanostructured materials toward energy conversion and storage. Nano-Micro Lett. 13, 49 (2021). https://doi.org/10.1007/s40820-020-00577-0
6. S. Tominaka, H. Nishizeko, J. Mizuno, T.J.E. Osaka, E. Science, Bendable fuel cells: on-chip fuel cell on a flexible polymer substrate. Energy Environ. Sci. 2, 1074-1077 (2009). https://doi.org/10.1039/b915389f

7. Z.M. Bhat, T. Ravikumar, M.C. Devendrachari, A.R. Kottaichamy, S.P. Shafi et al., Fuel exhaling fuel cell. J. Phys. Chem. Lett. 9, 388-392 (2018). https://doi.org/10.1021/acs. jpclett. $7 \mathrm{~b} 03100$

8. H. Zhao, A.F. Burke, Optimization of fuel cell system operating conditions for fuel cell vehicles. J. Power Sources 186, 408-416 (2009). https://doi.org/10.1016/j.jpowsour.2008.10. 032

9. W. Zong, C. Yang, L. Mo, Y. Ouyang, H. Guo et al., Elucidating dual-defect mechanism in rhenium disulfide nanosheets with multi-dimensional ion transport channels for ultrafast sodium storage. Nano Energy 77, 105189 (2020). https://doi. org/10.1016/j.nanoen.2020.105189

10. C. Wei, R.R. Rao, J. Peng, B. Huang, I.E.L. Stephens et al., Recommended practices and benchmark activity for hydrogen and oxygen electrocatalysis in water splitting and fuel cells. Adv. Mater. 31, 1806296 (2019). https://doi.org/10.1002/ adma.201806296

11. L. Huang, S. Zaman, Z. Wang, H. Niu, B. You et al., Synthesis and application of platinum-based hollow nanoframes for direct alcohol fuel cells. Acta Phys. Chim. Sin. 37(9), 2009035 (2020). https://doi.org/10.3866/pku.Whxb202009 035

12. A. Yoshino, The birth of the lithium-ion battery. Angew. Chem. Int. Ed. 51, 5798-5800 (2012). https://doi.org/10. 1002/anie.201105006

13. C.J. Yao, Z. Wu, J. Xie, F. Yu, W. Guo et al., Two-dimensional (2D) covalent organic framework as efficient cathode for binder-free lithium-ion battery. Chemsuschem 13, 24572463 (2020). https://doi.org/10.1002/cssc.201903007

14. E.W.-G. Diau, Next-generation solar cells and conversion of solar energy. ACS Energy Lett. 2, 334-335 (2017). https:// doi.org/10.1021/acsenergylett.6b00645

15. L. Yin, Y. Li, X. Yao, Y. Wang, L. Jia et al., MXenes for solar cells. Nano-Micro Lett. 13, 1-17 (2021). https://doi.org/10. 1007/s40820-021-00604-8

16. Q. Zhu, D. Zhao, M. Cheng, J. Zhou, K.A. Owusu et al., A new view of supercapacitors: integrated supercapacitors. Adv. Energy Mater. 9, 1901081 (2019). https://doi.org/10.1002/ aenm.201901081

17. B. Guo, R. Ma, Z. Li, S. Guo, J. Luo et al., Hierarchical $\mathrm{N}$-doped porous carbons for $\mathrm{Zn}$-air batteries and supercapacitors. Nano-Micro Lett. 12, 20 (2020). https://doi.org/10.1007/ s40820-019-0364-z

18. W. Zong, N. Chui, Z. Tian, Y. Li, C. Yang et al., Ultrafine MoP nanoparticle splotched nitrogen-doped carbon nanosheets enabling high-performance 3D-printed potassium-ion hybrid capacitors. Adv. Sci. 8, 2004142 (2021). https://doi.org/10.1002/advs.202004142

19. X. Duan, N. Pan, C. Sun, K. Zhang, X. Zhu et al., MOFderived Co-MOF, O-doped carbon as trifunctional electrocatalysts to enable highly efficient $\mathrm{Zn}$-air batteries and 
water-splitting. J. Energy Chem. 56, 290-298 (2021). https:// doi.org/10.1016/j.jechem.2020.08.007

20. J. Jiao, Y. Pan, B. Wang, W. Yang, S. Liu et al., Melamineassisted pyrolytic synthesis of bifunctional cobalt-based coreshell electrocatalysts for rechargeable zinc-air batteries. J. Energy Chem. 53, 364-371 (2021). https://doi.org/10.1016/j. jechem.2020.05.032

21. L. Li, Z.W. Chang, X.B. Zhang, Recent progress on the development of metal-air batteries. Adv. Sustain. Syst. 1, 1700036 (2017). https://doi.org/10.1002/adsu.201700036

22. W. Yu, W. Shang, P. Tan, B. Chen, Z. Wu et al., Toward a new generation of low cost, efficient, and durable metal-air flow batteries. J. Mater. Chem. A 7, 26744-26768 (2019). https:// doi.org/10.1039/C9TA10658H

23. J.L. Ma, F.L. Meng, Y. Yu, D.P. Liu, J.M. Yan et al., Prevention of dendrite growth and volume expansion to give high-performance aprotic bimetallic $\mathrm{Li}-\mathrm{Na}$ alloy- $\mathrm{O}_{2}$ batteries. Nat. Chem. 11, 64-70 (2019). https://doi.org/10.1038/ s41557-018-0166-9

24. J.-J. Xu, X.-B. Zhang, Li-air batteries: decouple to stabilize. Nat. Energy 2, 1-2 (2017). https://doi.org/10.1038/nenergy. 2017.133

25. Y.B. Yin, X.Y. Yang, Z.W. Chang, Y.H. Zhu, T. Liu et al., A water-/fireproof flexible lithium-oxygen battery achieved by synergy of novel architecture and multifunctional separator. Adv. Mater. 30, 1703791 (2018). https://doi.org/10.1002/ adma. 201703791

26. W. Zhang, L. Sun, J.M.V. Nsanzimana, X. Wang, Lithiation/delithiation synthesis of few layer silicene nanosheets for rechargeable $\mathrm{Li}-\mathrm{O}_{2}$ batteries. Adv. Mater. 30, 1705523 (2018). https://doi.org/10.1002/adma.201705523

27. J. Pan, Y.Y. Xu, H. Yang, Z. Dong, H. Liu et al., Advanced architectures and relatives of air electrodes in $\mathrm{Zn}$-air batteries. Adv. Sci. 5, 1700691 (2018). https://doi.org/10.1002/advs. 201700691

28. F.L. Meng, K.H. Liu, Y. Zhang, M.M. Shi, X.B. Zhang et al., Recent advances toward the rational design of efficient bifunctional air electrodes for rechargeable $\mathrm{Zn}$-air batteries. Small 14, 1703843 (2018). https://doi.org/10.1002/smll. 201703843

29. A.I. Douka, H. Yang, L. Huang, S. Zaman, T. Yue et al., Transition metal/carbon hybrids for oxygen electrocatalysis in rechargeable zinc-air batteries. EcoMat 3, 12067 (2020). https://doi.org/10.1002/eom2.12067

30. P. Cai, Y. Li, J. Chen, J. Jia, G. Wang et al., An asymmetric-electrolyte $\mathrm{Zn}$-air battery with ultrahigh power density and energy density. ChemElectroChem 5, 589-592 (2018). https://doi.org/10.1002/celc.201701269

31. X. Cai, L. Lai, J. Lin, Z. Shen, Recent advances in air electrodes for $\mathrm{Zn}$-air batteries: electrocatalysis and structural design. Mater. Horizons 4, 945-976 (2017). https://doi.org/ 10.1039/C7MH00358G

32. X. Han, X. Li, J. White, C. Zhong, Y. Deng et al., Metal-air batteries: from static to flow system. Adv. Energy Mater. 8, 1801396 (2018). https://doi.org/10.1002/aenm.201801396
33. L. Li, J. He, Y. Wang, X. Lv, X. Gu et al., Metal-organic frameworks: a promising platform for constructing non-noble electrocatalysts for the oxygen-reduction reaction. J. Mater. Chem. A 7, 1964-1988 (2019). https://doi.org/10.1039/ C8TA11704G

34. W. Fang, L. Huang, S. Zaman, Z. Wang, Y. Han et al., Recent progress on two-dimensional electrocatalysis. Chem. Res. Chin. Univ. 36, 611-621 (2020). https://doi.org/10.1007/ s40242-020-0182-3

35. S. Dou, X. Wang, S. Wang, Rational design of transition metal-based materials for highly efficient electrocatalysis. Small Methods 3, 1800211 (2019). https://doi.org/10.1002/ smtd.201800211

36. M.Y. Masoomi, A. Morsali, A. Dhakshinamoorthy, H. Garcia, Mixed-metal MOFs: unique opportunities in metal-organic framework (MOF) functionality and design. Angew. Chem. Int. Ed. 58, 15188-15205 (2019). https://doi.org/10.1002/ anie. 201902229

37. H. Zhong, K. Liu, Q. Zhang, F. Meng, D. Bao et al., Copper tetrazolate based metal-organic frameworks as highly efficient catalysts for artificially chemical and electrochemical $\mathrm{CO}_{2}$ conversion. Nano Select 1, 311-319 (2020). https://doi. org/10.1002/nano.202000041

38. F.X.L.I. Xamena, A. Abad, A. Corma, H. Garcia, MOFs as catalysts: activity, reusability and shape-selectivity of a Pdcontaining MOF. J. Catal. 250, 294-298 (2007). https://doi. org/10.1016/j.jcat.2007.06.004

39. S. Jin, How to effectively utilize MOFs for electrocatalysis. ACS Energy Lett. 4, 1443-1445 (2019). https://doi.org/10. 1021/acsenergylett.9b01134

40. S. Yang, L. Peng, S. Bulut, W.L. Queen, Recent advances of MOFs and MOF-derived materials in thermally driven organic transformations. Chem. Eur. J. 25, 2161-2178 (2019). https://doi.org/10.1002/chem.201803157

41. B.Y. Xia, Y. Yan, N. Li, H.B. Wu, X.W. Lou et al., A metalorganic framework-derived bifunctional oxygen electrocatalyst. Nat. Energy 1, 15006 (2016). https://doi.org/10.1038/ nenergy.2015.6

42. X.F. Lu, B.Y. Xia, S.Q. Zang, X.W.D. Lou, Metal-organic frameworks based electrocatalysts for the oxygen reduction reaction. Angew. Chem. Int. Ed. 59, 4634-4650 (2020). https://doi.org/10.1002/anie.201910309

43. X. Tian, X.F. Lu, B.Y. Xia, X.W. Lou, Advanced electrocatalysts for the oxygen reduction reaction in energy conversion technologies. Joule 4, 45-68 (2020). https://doi.org/10. 1016/j.joule.2019.12.014

44. J. Pan, X.L. Tian, S. Zaman, Z. Dong, H. Liu et al., Recent progress on transition metal oxides as bifunctional catalysts for lithium-air and zinc-air batteries. Batteries Supercaps 2, 336-347 (2018). https://doi.org/10.1002/batt.201800082

45. Y. Xu, P. Deng, G. Chen, J. Chen, Y. Yan et al., 2D nitrogendoped carbon nanotubes/graphene hybrid as bifunctional oxygen electrocatalyst for long-life rechargeable $\mathrm{Zn}$-air batteries. Adv. Funct. Mater. 30, 1906081 (2019). https://doi.org/10. 1002/adfm.201906081 
46. H. Yang, X. Han, A.I. Douka, L. Huang, L. Gong et al., Advanced oxygen electrocatalysis in energy conversion and storage. Adv. Funct. Mater. 2007602 (2020). https://doi.org/ 10.1002/adfm.202007602

47. X. Liu, T. Yue, K. Qi, Y. Qiu, B.Y. Xia et al., Metal-organic framework membranes: from synthesis to electrocatalytic applications. Chin. Chem. Lett. 31, 2189-2201 (2020). https://doi.org/10.1016/j.cclet.2019.12.009

48. Y. Yan, Y. Xu, B. Zhao, Y. Xu, Y. Gao et al., Bifunctional nickel ferrite-decorated carbon nanotube arrays as freestanding air electrode for rechargeable $\mathrm{Zn}$-air batteries. J. Mater. Chem. A 8, 5070-5077 (2020). https://doi.org/10. 1039/d0ta00554a

49. A.I. Douka, Y. Xu, H. Yang, S. Zaman, Y. Yan et al., A zeolitic-imidazole frameworks-derived interconnected macroporous carbon matrix for efficient oxygen electrocatalysis in rechargeable zinc-air batteries. Adv. Mater. 32, 2002170 (2020). https://doi.org/10.1002/adma.202002170

50. G. Chen, Y. Xu, L. Huang, A.I. Douka, B.Y. Xia, Continuous nitrogen-doped carbon nanotube matrix for boosting oxygen electrocatalysis in rechargeable $\mathrm{Zn}$-air batteries. J. Energy Chem. 55, 183-189 (2021). https://doi.org/10. 1016/j.jechem.2020.07.012

51. S. Zaman, L. Huang, A.I. Douka, H. Yang, B. You et al., Oxygen reduction electrocatalysts toward practical fuel cells: progress and perspectives. Angew. Chem. Int. Ed. 2016977 (2021). https://doi.org/10.1002/anie.202016977

52. S. Głowniak, B. Szczęśniak, J. Choma, M. Jaroniec, Mechanochemistry: toward green synthesis of metal-organic frameworks. Mater. Today (In press, 2021). https://doi.org/ 10.1016/j.mattod.2021.01.008

53. Z. Liang, H. Guo, G. Zhou, K. Guo, B. Wang et al., Metalorganic-framework-supported molecular electrocatalysis for the oxygen reduction reaction. Angew. Chem. Int. Ed. Engl. 60, 8472-8476 (2021). https://doi.org/10.1002/anie.20201 6024

54. Y. Yang, X. Zhang, S. Kanchanakungwankul, Z. Lu, H. Noh et al., Unexpected "spontaneous" evolution of catalytic, MOF-supported single $\mathrm{Cu}(\mathrm{II})$ cations to catalytic, MOFsupported $\mathrm{Cu}(0)$ nanoparticles. J. Am. Chem. Soc. 142, 21169-21177 (2020). https://doi.org/10.1021/jacs.0c10367

55. S. Bai, X. Liu, K. Zhu, S. Wu, H. Zhou, Metal-organic framework-based separator for lithium-sulfur batteries. Nat. Energy 1, 16094 (2016). https://doi.org/10.1038/nenergy. 2016.94

56. X. Zhu, C. Hu, R. Amal, L. Dai, X. Lu, Heteroatom-doped carbon catalysts for zinc-air batteries: progress, mechanism, and opportunities. Energy Environ. Sci. 13, 4536-4563 (2020). https://doi.org/10.1039/D0EE02800B

57. J. Fu, Z.P. Cano, M.G. Park, A. Yu, M. Fowler et al., Electrically rechargeable zinc-air batteries: progress, challenges, and perspectives. Adv. Mater. 29, 1604685 (2017). https://doi.org/ 10.1002/adma.201604685

58. M. Wu, Y. Wang, Z. Wei, L. Wang, M. Zhuo et al., Ternary doped porous carbon nanofibers with excellent ORR and
OER performance for zinc-air batteries. J. Mater. Chem. A 6, 10918-10925 (2018). https://doi.org/10.1039/C8TA02416B

59. Y. Li, H. Dai, Recent advances in zinc-air batteries. Chem. Soc. Rev. 43, 5257-5275 (2014). https://doi.org/10.1039/ C4CS00015C

60. S. Kuyuldar, D.T. Genna, C. Burda, On the potential for nanoscale metal-organic frameworks for energy applications. J. Mater. Chem. A 7, 21545-21576 (2019). https://doi.org/10. 1039/c9ta09896h

61. D. Ren, J. Ying, M. Xiao, Y.P. Deng, J. Ou et al., Hierarchically porous multimetal-based carbon nanorod hybrid as an efficient oxygen catalyst for rechargeable zinc-air batteries. Adv. Funct. Mater. 30, 1908167 (2019). https://doi.org/10. 1002/adfm.201908167

62. K. Ge, S. Sun, Y. Zhao, K. Yang, S. Wang et al., Facile synthesis of two-dimensional Fe/Co metal-organic framework for efficient oxygen evolution electrocatalysis. Angew. Chem. Int. Ed. 2102632 (2021). https://doi.org/10.1002/anie.202102632

63. Z.-F. Huang, J. Song, Y. Du, S. Xi, S. Dou et al., Chemical and structural origin of lattice oxygen oxidation in Co-Zn oxyhydroxide oxygen evolution electrocatalysts. Nat. Energy 4, 329-338 (2019). https://doi.org/10.1038/ s41560-019-0355-9

64. J. Liu, Y. Gao, X. Tang, K. Zhan, B. Zhao et al., Metalorganic framework-derived hierarchical ultrathin CoP nanosheets for overall water splitting. J. Mater. Chem. A 8, 19254-19261 (2020). https://doi.org/10.1039/d0ta07616c

65. J.M.V. Nsanzimana, L. Gong, R. Dangol, V. Reddu, V. Jose et al., Tailoring of metal boride morphology via anion for efficient water oxidation. Adv. Energy Mater. 9, 1901503 (2019). https://doi.org/10.1002/aenm.201901503

66. Y. Xu, Y. Yan, T. He, K. Zhan, J. Yang et al., Supercritical $\mathrm{CO}_{2}$-Assisted synthesis of $\mathrm{NiFe}_{2} \mathrm{O}_{4} /$ vertically-aligned carbon nanotube arrays hybrid as a bifunctional electrocatalyst for efficient overall water splitting. Carbon 145, 201-208 (2019). https://doi.org/10.1016/j.carbon.2019.01.011

67. Q. Guan, Y. Li, X. Bi, J. Yang, J. Zhou et al., Dendrite-free flexible fiber-shaped Zn battery with long cycle life in water and air. Adv. Energy Mater. 9, 1901434 (2019). https://doi. org/10.1002/aenm.201901434

68. B. Zhu, D. Xia, R. Zou, Metal-organic frameworks and their derivatives as bifunctional electrocatalysts. Coord. Chem. Rev. 376, 430-448 (2018). https://doi.org/10.1016/j.ccr.2018. 07.020

69. R. Zhao, Z. Liang, R. Zou, Q. Xu, Metal-organic frameworks for batteries. Joule 2, 2235-2259 (2018). https://doi.org/10. 1016/j.joule.2018.09.019

70. T. Mehtab, G. Yasin, M. Arif, M. Shakeel, R.M. Korai et al., Metal-organic frameworks for energy storage devices: batteries and supercapacitors. J. Energy Storage 21, 632-646 (2019). https://doi.org/10.1016/j.est.2018.12.025

71. L. Zou, C.C. Hou, Q. Wang, Y.S. Wei, Z. Liu et al., A honeycomb-like bulk superstructure of carbon nanosheets for electrocatalysis and energy storage. Angew. Chem. Int. Ed. 59, 19627-19632 (2020). https://doi.org/10.1002/anie.20200 4737 
72. X. Wang, X.Y. Li, C.B. Ouyang, Z. Li, S. Dou et al., Nonporous MOF-derived dopant-free mesoporous carbon as an efficient metal-free electrocatalyst for the oxygen reduction reaction. J. Mater. Chem. A 4, 9370-9374 (2016). https://doi. org/10.1039/C6TA03015G

73. M. Zhang, D. Wu, Y. Ye, L. Wu, Z. Yao et al., Thermal conversion of MOF@MOF: synthesis of an N-doped carbon material with excellent ORR performance. ChemPlusChem 83, 1044-1051 (2018). https://doi.org/10.1002/cplu.20180 0392

74. A. Aijaz, N. Fujiwara, Q. Xu, From metal-organic framework to nitrogen-decorated nanoporous carbons: high $\mathrm{CO}_{2}$ uptake and efficient catalytic oxygen reduction. J. Am. Chem. Soc. 136, 6790-6793 (2014). https://doi.org/10. 1021/ja5003907

75. W. Zhang, Z.Y. Wu, H.L. Jiang, S.H. Yu, Nanowire-directed templating synthesis of metal-organic framework nanofibers and their derived porous doped carbon nanofibers for enhanced electrocatalysis. J. Am. Chem. Soc. 136, 1438514388 (2014). https://doi.org/10.1021/ja5084128

76. H.X. Zhong, J. Wang, Y.W. Zhang, W.L. Xu, W. Xing et al., ZIF-8 derived graphene-based nitrogen-doped porous carbon sheets as highly efficient and durable oxygen reduction electrocatalysts. Angew. Chem. Int. Ed. 53, 14235-14239 (2014). https://doi.org/10.1002/anie.201408990

77. X. Wang, J. Zhou, H. Fu, W. Li, X. Fan et al., MOF derived catalysts for electrochemical oxygen reduction. J. Mater. Chem. A 2, 14064-14070 (2014). https://doi.org/10.1039/ c4ta01506a

78. M. Wu, K. Wang, M. Yi, Y. Tong, Y. Wang et al., A facile activation strategy for an MOF-derived metal-free oxygen reduction reaction catalyst: direct access to optimized pore structure and nitrogen species. ACS Catal. 7, 6082-6088 (2017). https://doi.org/10.1021/acscatal.7b01649

79. L. Zhang, Z. Su, F. Jiang, L. Yang, J. Qian et al., Highly graphitized nitrogen-doped porous carbon nanopolyhedra derived from ZIF-8 nanocrystals as efficient electrocatalysts for oxygen reduction reactions. Nanoscale 6, 6590-6602 (2014). https://doi.org/10.1039/c4nr00348a

80. M. Yang, X. Hu, Z. Fang, L. Sun, Z. Yuan et al., Bifunctional MOF-derived carbon photonic crystal architectures for advanced $\mathrm{Zn}$-air and Li-S batteries: highly exposed graphitic nitrogen matters. Adv. Funct. Mater. 27, 1701971 (2017). https://doi.org/10.1002/adfm.201701971

81. L. Chai, L. Zhang, X. Wang, L. Xu, C. Han et al., Bottom-up synthesis of MOF-derived hollow N-doped carbon materials for enhanced ORR performance. Carbon 146, 248-256 (2019). https://doi.org/10.1016/j.carbon.2019.02.006

82. P. Zhang, F. Sun, Z. Xiang, Z. Shen, J. Yun et al., ZIF-derived in situ nitrogen-doped porous carbons as efficient metal-free electrocatalysts for oxygen reduction reaction. Energy Environ. Sci. 7, 442-450 (2014). https://doi.org/10.1039/c3ee4 2799d

83. Y. Lv, L. Yang, D. Cao, Nitrogen and fluorine-codoped porous carbons as efficient metal-free electrocatalysts for oxygen reduction reaction in fuel cells. ACS Appl. Mater.
Interfaces 9, 32859-32867 (2017). https://doi.org/10.1021/ acsami.7b11371

84. J. Shui, C. Chen, L. Grabstanowicz, D. Zhao, D.J. Liu, Highly efficient nonprecious metal catalyst prepared with metalorganic framework in a continuous carbon nanofibrous network. Proc. Natl. Acad. Sci. USA 112, 10629-10634 (2015). https://doi.org/10.1073/pnas.1507159112

85. Y. Qian, Z. Hu, X. Ge, S. Yang, Y. Peng et al., A metal-free ORR/OER bifunctional electrocatalyst derived from metalorganic frameworks for rechargeable Zn-Air batteries. Carbon 111, 641-650 (2017). https://doi.org/10.1016/j.carbon.2016. 10.046

86. J. Li, Y. Chen, Y. Tang, S. Li, H. Dong et al., Metal-organic framework templated nitrogen and sulfur co-doped porous carbons as highly efficient metal-free electrocatalysts for oxygen reduction reactions. J. Mater. Chem. A 2, 6316-6319 (2014). https://doi.org/10.1039/c3ta15335e

87. X. Wen, Q. Zhang, J. Guan, Applications of metal-organic framework-derived materials in fuel cells and metal-air batteries. Coord. Chem. Rev. 409, 213214 (2020). https://doi. org/10.1016/j.ccr.2020.213214

88. J. Song, C. Wei, Z.F. Huang, C. Liu, L. Zeng et al., A review on fundamentals for designing oxygen evolution electrocatalysts. Chem. Soc. Rev. 49, 2196-2214 (2020). https://doi.org/ $10.1039 / \mathrm{c} 9 \mathrm{cs} 00607 \mathrm{a}$

89. C. Lu, R. Fang, X. Chen, Single-atom catalytic materials for advanced battery systems. Adv. Mater. 32, 1906548 (2020). https://doi.org/10.1002/adma.201906548

90. Q. Wang, X. Huang, Z.L. Zhao, M. Wang, B. Xiang et al., Ultrahigh-loading of Ir single atoms on $\mathrm{NiO}$ matrix to dramatically enhance oxygen evolution reaction. J. Am. Chem. Soc. 142, 7425-7433 (2020). https://doi.org/10.1021/jacs. $9 b 12642$

91. K. Chen, S. Kim, M. Je, H. Choi, Z. Shi et al., Ultrasonic plasma engineering toward facile synthesis of singleatom $\mathrm{M}-\mathrm{N}_{4} / \mathrm{N}$-doped carbon $(\mathrm{M}=\mathrm{Fe}, \mathrm{Co})$ as superior oxygen electrocatalyst in rechargeable zinc-air batteries. Nano-Micro Lett. 13, 60 (2021). https://doi.org/10.1007/ s40820-020-00581-4

92. H. Zhang, G. Liu, L. Shi, J. Ye, Single-atom catalysts: emerging multifunctional materials in heterogeneous catalysis. Adv. Energy Mater. 8, 1701343 (2018). https://doi.org/10.1002/ aenm.201701343

93. L. Jiao, Y. Wang, H.L. Jiang, Q. Xu, Metal-organic frameworks as platforms for catalytic applications. Adv. Mater. 30, 1703663 (2018). https://doi.org/10.1002/adma.201703663

94. L. Jiao, H.-L. Jiang, Metal-organic-framework-based singleatom catalysts for energy applications. Chem 5, 786-804 (2019). https://doi.org/10.1016/j.chempr.2018.12.011

95. Y. Lin, P. Liu, E. Velasco, G. Yao, Z. Tian et al., Fabricating single-atom catalysts from chelating metal in open frameworks. Adv. Mater. 31, 1808193 (2019). https://doi.org/10. 1002/adma.201808193

96. X. Han, X. Ling, Y. Wang, T. Ma, C. Zhong et al., Generation of nanoparticle, atomic-cluster, and single-atom cobalt catalysts from zeolitic imidazole frameworks by spatial isolation 
and their use in zinc-air batteries. Angew. Chem. Int. Ed. 58, 5359-5364 (2019). https://doi.org/10.1002/anie.201901109

97. P. Yin, T. Yao, Y. Wu, L. Zheng, Y. Lin et al., Single cobalt atoms with precise $\mathrm{N}$-coordination as superior oxygen reduction reaction catalysts. Angew. Chem. Int. Ed. 55, 10800-10805 (2016). https://doi.org/10.1002/anie.20160 4802

98. Y. Qu, Z. Li, W. Chen, Y. Lin, T. Yuan et al., Direct transformation of bulk copper into copper single sites via emitting and trapping of atoms. Nat. Catal. 1, 781-786 (2018). https://doi.org/10.1038/s41929-018-0146-X

99. W. Sun, L. Du, Q. Tan, J. Zhou, Y. Hu et al., Engineering of nitrogen coordinated single cobalt atom moieties for oxygen electroreduction. ACS Appl. Mater. Interfaces 11, 41258-41266 (2019). https://doi.org/10.1021/acsami.9b118 30

100. Y. Chen, R. Gao, S. Ji, H. Li, K. Tang et al., Atomic-level modulation of electronic density at cobalt single-atom sites derived from metal-organic frameworks: enhanced oxygen reduction performance. Angew. Chem. Int. Ed. 60, 32123221 (2020). https://doi.org/10.1002/anie.202012798

101. H. Liu, M.Q. Wang, Z.Y. Chen, H. Chen, M.W. Xu et al., Design and synthesis of Co-N-C porous catalyst derived from metal organic complexes for highly effective ORR. Dalton Trans. 46, 15646-15650 (2017). https://doi.org/10.1039/ c7dt03279j

102. S. Dilpazir, H. He, Z. Li, M. Wang, P. Lu et al., Cobalt single atoms immobilized $\mathrm{N}$-doped carbon nanotubes for enhanced bifunctional catalysis toward oxygen reduction and oxygen evolution reactions. ACS Appl. Energy Mater. 1, 3283-3291 (2018). https://doi.org/10.1021/acsaem.8b00490

103. W. Zang, A. Sumboja, Y. Ma, H. Zhang, Y. Wu et al., Single Co atoms anchored in porous $\mathrm{N}$-doped carbon for efficient zinc-air battery cathodes. ACS Catal. 8, 8961-8969 (2018). https://doi.org/10.1021/acscatal.8b02556

104. D. Ji, L. Fan, L. Li, S. Peng, D. Yu et al., Atomically transition metals on self-supported porous carbon flake arrays as binder-free air cathode for wearable zinc-air batteries. Adv. Mater. 31, 1808267 (2019). https://doi.org/10.1002/adma. 201808267

105. Y. Li, R. Cao, L. Li, X. Tang, T. Chu et al., Simultaneously integrating single atomic cobalt sites and $\mathrm{Co}_{9} \mathrm{~S}_{8}$ nanoparticles into hollow carbon nanotubes as trifunctional electrocatalysts for $\mathrm{Zn}$-air batteries to drive water splitting. Small 16, 1906735 (2020). https://doi.org/10.1002/smll.201906735

106. Y. Chen, S. Ji, S. Zhao, W. Chen, J. Dong et al., Enhanced oxygen reduction with single-atomic-site iron catalysts for a zinc-air battery and hydrogen-air fuel cell. Nat. Commun. 9, 5422 (2018). https://doi.org/10.1038/s41467-018-07850-2

107. Y. Zhu, B. Zhang, X. Liu, D.W. Wang, D.S. Su, Unravelling the structure of electrocatalytically active $\mathrm{Fe}-\mathrm{N}$ complexes in carbon for the oxygen reduction reaction. Angew. Chem. Int. Ed. 53, 10673-10677 (2014). https://doi.org/10.1002/anie. 201405314

108. X. Gong, J. Zhu, J. Li, R. Gao, Q. Zhou et al., Self-templated hierarchically porous carbon nanorods embedded with atomic
$\mathrm{Fe}-\mathrm{N}_{4}$ active sites as efficient oxygen reduction electrocatalysts in Zn-air batteries. Adv. Funct. Mater. 31, 2008085 (2020). https://doi.org/10.1002/adfm.202008085

109. L. Ma, S. Chen, Z. Pei, Y. Huang, G. Liang et al., Single-site active iron-based bifunctional oxygen catalyst for a compressible and rechargeable zinc-air battery. ACS Nano 12, 1949-1958 (2018). https://doi.org/10.1021/acsnano.7b09064

110. Y. Han, Q.K. Li, K. Ye, Y. Luo, J. Jiang et al., Impact of active site density on oxygen reduction reactions using monodispersed $\mathrm{Fe}-\mathrm{N}-\mathrm{C}$ single-atom catalysts. ACS Appl. Mater. Interfaces 12, 15271-15278 (2020). https://doi.org/10.1021/ acsami.0c01206

111. J. Han, X. Meng, L. Lu, J. Bian, Z. Li et al., Single-atom $\mathrm{Fe}-\mathrm{N}_{x}-\mathrm{C}$ as an efficient electrocatalyst for zinc-air batteries. Adv. Funct. Mater. 29, 1808872 (2019). https://doi.org/10. 1002/adfm.201808872

112. C.C. Hou, L. Zou, L. Sun, K. Zhang, Z. Liu et al., Singleatom iron catalysts on overhang-eave carbon cages for highperformance oxygen reduction reaction. Angew. Chem. Int. Ed. 132, 7454-7459 (2020). https://doi.org/10.1002/anie. 202002665

113. Z. Lin, H. Huang, L. Cheng, Y. Yang, R. Zhang et al., Atomically dispersed $\mathrm{Mn}$ within carbon frameworks as high-performance oxygen reduction electrocatalysts for zinc-air battery. ACS Sustain. Chem. Eng. 8, 427-434 (2019). https://doi.org/ 10.1021/acssuschemeng.9b05713

114. X. Han, T. Zhang, W. Chen, B. Dong, G. Meng et al., Mn- $\mathrm{N}_{4}$ oxygen reduction electrocatalyst: operando investigation of active sites and high performance in zinc-air battery. Adv. Energy Mater. 11, 2002753 (2020). https://doi.org/10.1002/ aenm.202002753

115. J. Wang, F. Xu, H. Jin, Y. Chen, Y. Wang, Non-noble metalbased carbon composites in hydrogen evolution reaction: fundamentals to applications. Adv. Mater. 29, 1605838 (2017). https://doi.org/10.1002/adma.201605838

116. S. Bhattacharyya, C. Das, T.K. Maji, MOF derived carbon based nanocomposite materials as efficient electrocatalysts for oxygen reduction and oxygen and hydrogen evolution reactions. RSC Adv. 8, 26728-26754 (2018). https://doi.org/ $10.1039 / \mathrm{c} 8 \mathrm{ra05102 \textrm {j }}$

117. S. Liu, Z. Wang, S. Zhou, F. Yu, M. Yu et al., Metal-organicframework-derived hybrid carbon nanocages as a bifunctional electrocatalyst for oxygen reduction and evolution. Adv. Mater. 29, 1700874 (2017). https://doi.org/10.1002/adma. 201700874

118. X. Chen, N. Wang, K. Shen, Y. Xie, Y. Tan et al., MOFderived isolated $\mathrm{Fe}$ atoms implanted in $\mathrm{N}$-doped 3D hierarchical carbon as an efficient ORR electrocatalyst in both alkaline and acidic media. ACS Appl. Mater. Interfaces 11, 25976-25985 (2019). https://doi.org/10.1021/acsami.9b074 36

119. M. Qiao, Y. Wang, X. Mamat, A. Chen, G. Zou et al., Rational design of hierarchical, porous, Co-supported, $\mathrm{N}$-doped carbon architectures as electrocatalyst for oxygen reduction. Chemsuschem 13, 741-748 (2020). https://doi.org/ $10.1002 /$ cssc. 201903053 
120. M. Zhang, Q. Dai, H. Zheng, M. Chen, L. Dai, Novel MOFderived Co@N-C bifunctional catalysts for highly efficient Zn-air batteries and water splitting. Adv. Mater. 30, 1705431 (2018). https://doi.org/10.1002/adma.201705431

121. W. Xie, J. Li, Y. Song, S. Li, J. Li et al., Hierarchical carbon microtube@nanotube core-shell structure for highperformance oxygen electrocatalysis and $\mathrm{Zn}$-air battery. Nano-Micro Lett. 12, 1-14 (2020). https://doi.org/10.1007/ s40820-020-00435-z

122. Q. Niu, B. Chen, J. Guo, J. Nie, X. Guo et al., Flexible, porous, and metal-heteroatom-doped carbon nanofibers as efficient ORR electrocatalysts for Zn-air battery. Nano-Micro Lett. 11, 8 (2019). https://doi.org/10.1007/ s40820-019-0238-4

123. J. Wu, L. Hu, N. Wang, Y. Li, D. Zhao et al., Surface confinement assisted synthesis of nitrogen-rich hollow carbon cages with Co nanoparticles as breathable electrodes for $\mathrm{Zn-air}$ batteries. Appl. Catal. B-Environ. 254, 55-65 (2019). https://doi.org/10.1016/j.apcatb.2019.04.064

124. Q. Lai, J. Zhu, Y. Zhao, Y. Liang, J. He et al., MOF-based metal-doping-induced synthesis of hierarchical porous $\mathrm{Cu}-$ N/C oxygen reduction electrocatalysts for $\mathrm{Zn}$-air batteries. Small 13, 1700740 (2017). https://doi.org/10.1002/smll. 201700740

125. X. Zheng, Y. Cao, D. Liu, M. Cai, J. Ding et al., Bimetallic metal-organic-framework/reduced graphene oxide composites as bifunctional electrocatalysts for rechargeable $\mathrm{Zn}$-air batteries. ACS Appl. Mater. Interfaces 11, 15662-15669 (2019). https://doi.org/10.1021/acsami.9b02859

126. S.S. Shinde, C.H. Lee, J.-Y. Jung, N.K. Wagh, S.-H. Kim et al., Unveiling dual-linkage 3D hexaiminobenzene metalorganic frameworks towards long-lasting advanced reversible Zn-air batteries. Energy Environ. Sci. 12, 727-738 (2019). https://doi.org/10.1039/c8ee02679c

127. Z. Wang, H. Jin, T. Meng, K. Liao, W. Meng et al., Fe, $\mathrm{Cu}$-coordinated ZIF-derived carbon framework for efficient oxygen reduction reaction and zinc-air batteries. Adv. Funct. Mater. 28, 1802596 (2018). https://doi.org/10.1002/ adfm.201802596

128. A. Pendashteh, S.M.F. Vilela, I. Krivtsov, D. Ávila-Brande, J. Palma et al., Bimetal zeolitic imidazolate framework (ZIF-9) derived nitrogen-doped porous carbon as efficient oxygen electrocatalysts for rechargeable $\mathrm{Zn}$-air batteries. J. Power Sources 427, 299-308 (2019). https://doi.org/10. 1016/j.jpowsour.2019.04.074

129. C. Zhang, H. Yang, D. Zhong, Y. Xu, Y. Wang et al., A yolk-shell structured metal-organic framework with encapsulated iron-porphyrin and its derived bimetallic nitrogendoped porous carbon for an efficient oxygen reduction reaction. J. Mater. Chem. A 8, 9536-9544 (2020). https://doi. org/10.1039/d0ta00962h

130. Y. Jiang, Y.P. Deng, R. Liang, J. Fu, R. Gao et al., d-Orbital steered active sites through ligand editing on heterometal imidazole frameworks for rechargeable zinc-air battery. Nat. Commun. 11, 5858 (2020). https://doi.org/10.1038/ s41467-020-19709-6
131. B. Chen, X. He, F. Yin, H. Wang, D.-J. Liu et al., MOCo@N-doped carbon $(\mathrm{M}=\mathrm{Zn}$ or $\mathrm{Co})$ : vital roles of inactive $\mathrm{Zn}$ and highly efficient activity toward oxygen reduction/evolution reactions for rechargeable $\mathrm{Zn}$-air battery. Adv. Funct. Mater. 27, 1700795 (2017). https://doi.org/10. 1002/adfm.201700795

132. T. Wang, Z. Kou, S. Mu, J. Liu, D. He et al., 2D dual-metal zeolitic-imidazolate-framework-(ZIF)-derived bifunctional air electrodes with ultrahigh electrochemical properties for rechargeable zinc-air batteries. Adv. Funct. Mater. 28, 1705048 (2018). https://doi.org/10.1002/adfm.201705048

133. Y. Xu, Z. Huang, B. Wang, Z. Liang, C. Zhang et al., A two-dimensional multi-shelled metal-organic framework and its derived bimetallic $\mathrm{N}$-doped porous carbon for electrocatalytic oxygen reduction. Chem. Commun. 55, 1480514808 (2019). https://doi.org/10.1039/c9cc08250f

134. J. Tan, T. Thomas, J. Liu, L. Yang, L. Pan et al., Rapid microwave-assisted preparation of high-performance bifunctional $\mathrm{Ni}_{3} \mathrm{Fe} / \mathrm{Co}-\mathrm{N}-\mathrm{C}$ for rechargeable $\mathrm{Zn}$-air battery. Chem. Eng. J. 395, 125151 (2020). https://doi.org/10. 1016/j.cej.2020.125151

135. K. Li, W. Chen, Recent progress in high-entropy alloys for catalysts: synthesis, applications, and prospects. Mater. Today Energy 100638 (2021). https://doi.org/10.1016/j. mtener.2021.100638

136. C. Li, M. Wu, R. Liu, High-performance bifunctional oxygen electrocatalysts for zinc-air batteries over mesoporous $\mathrm{Fe} / \mathrm{Co}-\mathrm{N}-\mathrm{C}$ nanofibers with embedding FeCo alloy nanoparticles. Appl. Catal. B-Environ. 244, 150-158 (2019). https://doi.org/10.1016/j.apcatb.2018.11.039

137. W. Niu, Y. Yang, Amorphous MOF introduced N-doped graphene: an efficient and versatile electrocatalyst for zincair battery and water splitting. ACS Appl. Energy Mater. 1, 2440-2445 (2018). https://doi.org/10.1021/acsaem.8b005 94

138. L. Yang, S. Feng, G. Xu, B. Wei, L. Zhang, Electrospun MOF-based FeCo nanoparticles embedded in nitrogen-doped mesoporous carbon nanofibers as an efficient bifunctional catalyst for oxygen reduction and oxygen evolution reactions in zinc-air batteries. ACS Sustain. Chem. Eng. 7, 5462-5475 (2019). https://doi.org/10.1021/acssuschemeng.8b06624

139. C.C. Hou, L. Zou, Q. Xu, A hydrangea-like superstructure of open carbon cages with hierarchical porosity and highly active metal sites. Adv. Mater. 31, 1904689 (2019). https:// doi.org/10.1002/adma.201904689

140. M. Wu, B. Guo, A. Nie, R. Liu, Tailored architectures of FeNi alloy embedded in $\mathrm{N}$-doped carbon as bifunctional oxygen electrocatalyst for rechargeable zinc-air battery. J. Colloid Interface Sci. 561, 585-592 (2020). https://doi.org/10.1016/j. jcis.2019.11.033

141. D. Chen, Z. Li, Y. Zhou, X. Ma, H. Lin et al., Fe ${ }_{3}$ Pt intermetallic nanoparticles anchored on $\mathrm{N}$-doped mesoporous carbon for the highly efficient oxygen reduction reaction. Chem. Commun. 56, 4898-4901 (2020). https://doi.org/10. 1039/d0cc00895h 
142. Q. Shao, J. Liu, Q. Wu, Q. Li, H.-G. Wang et al., In situ coupling strategy for anchoring monodisperse $\mathrm{Co}_{9} \mathrm{~S}_{8}$ nanoparticles on $\mathrm{S}$ and $\mathrm{N}$ dual-doped graphene as a bifunctional electrocatalyst for rechargeable Zn-air battery. Nano-Micro Lett. 11, 4 (2019). https://doi.org/10.1007/s40820-018-0231-3

143. Y. Tian, L. Xu, M. Li, D. Yuan, X. Liu et al., Interface engineering of $\mathrm{CoS} / \mathrm{CoO} @ \mathrm{~N}$-doped graphene nanocomposite for high-performance rechargeable $\mathrm{Zn}$-air batteries. Nano-Micro Lett. 13, 15 (2020). https://doi.org/10.1007/ s40820-020-00526-x

144. D. Adekoya, S. Qian, X. Gu, W. Wen, D. Li et al., DFTguided design and fabrication of carbon-nitride-based materials for energy storage devices: a review. Nano-Micro Lett. 13, 44 (2020). https://doi.org/10.1007/s40820-020-00522-1

145. S.L. Zhang, B.Y. Guan, X.F. Lu, S. Xi, Y. Du et al., Metal atom-doped $\mathrm{Co}_{3} \mathrm{O}_{4}$ hierarchical nanoplates for electrocatalytic oxygen evolution. Adv. Mater. 32, 2002235 (2020). https://doi.org/10.1002/adma.202002235

146. S. Chen, S. Chen, B. Zhang, J. Zhang, Bifunctional oxygen electrocatalysis of N, S-codoped porous carbon with interspersed hollow $\mathrm{CoO}$ nanoparticles for rechargeable $\mathrm{Zn}$-air batteries. ACS Appl. Mater. Interfaces 11, 16720-16728 (2019). https://doi.org/10.1021/acsami.9b02819

147. S. Dou, X. Li, L. Tao, J. Huo, S. Wang, Cobalt nanoparticleembedded carbon nanotube/porous carbon hybrid derived from MOF-encapsulated $\mathrm{Co}_{3} \mathrm{O}_{4}$ for oxygen electrocatalysis. Chem. Commun. 52, 9727-9730 (2016). https://doi.org/10. 1039/C6CC05244D

148. Z. Guo, F. Wang, Y. Xia, J. Li, A.G. Tamirat et al., In situ encapsulation of core-shell-structured $\mathrm{Co} @ \mathrm{Co}_{3} \mathrm{O}_{4}$ into nitrogen-doped carbon polyhedra as a bifunctional catalyst for rechargeable Zn-air batteries. J. Mater. Chem. A 6, 14431453 (2018). https://doi.org/10.1039/c7ta09958d

149. D. Ji, L. Fan, L. Tao, Y. Sun, M. Li et al., The Kirkendall effect for engineering oxygen vacancy of hollow $\mathrm{Co}_{3} \mathrm{O}_{4}$ nanoparticles toward high-performance portable zinc-air batteries. Angew. Chem. Int. Ed. 58, 13840-13844 (2019). https://doi. org/10.1002/anie.201908736

150. H. Liu, Z. Mai, X. Xu, Y. Wang, A Co-MOF-derived oxygenvacancy-rich $\mathrm{Co}_{3} \mathrm{O}_{4}$-based composite as a cathode material for hybrid $\mathrm{Zn}$ batteries. Dalton Trans. 49, 2880-2887 (2020). https://doi.org/10.1039/c9dt04682h

151. N. Sikdar, B. Konkena, J. Masa, W. Schuhmann, T.K. Maji, $\mathrm{Co}_{3} \mathrm{O}_{4} @ \mathrm{Co} / \mathrm{NCNT}$ nanostructure derived from a dicyanamide-based metal-organic framework as an efficient bifunctional electrocatalyst for oxygen reduction and evolution reactions. Chem. Eur. J. 23, 18049-18056 (2017). https://doi. org/10.1002/chem.201704211

152. T. Singh, C. Das, N. Bothra, N. Sikdar, S. Das et al., MOF derived $\mathrm{Co}_{3} \mathrm{O}_{4} @ \mathrm{Co} / \mathrm{NCNT}$ nanocomposite for electrochemical hydrogen evolution, flexible zinc-air batteries, and overall water splitting. Inorg. Chem. 59, 3160-3170 (2020). https:// doi.org/10.1021/acs.inorgchem.9b03516

153. J.-T. Ren, G.-G. Yuan, C.-C. Weng, Z.-Y. Yuan, Rationally designed $\mathrm{Co}_{3} \mathrm{O}_{4}-\mathrm{C}$ nanowire arrays on $\mathrm{Ni}$ foam derived from metal organic framework as reversible oxygen evolution electrodes with enhanced performance for $\mathrm{Zn}$-air batteries. ACS Sustain. Chem. Eng. 6, 707-718 (2017). https://doi.org/ 10.1021/acssuschemeng.7b03034

154. C. Guan, A. Sumboja, H. Wu, W. Ren, X. Liu et al., Hollow $\mathrm{Co}_{3} \mathrm{O}_{4}$ nanosphere embedded in carbon arrays for stable and flexible solid-state zinc-air batteries. Adv. Mater. 29, 1704117 (2017). https://doi.org/10.1002/adma.201704117

155. Y. Zhong, Z. Pan, X. Wang, J. Yang, Y. Qiu et al., Hierarchical $\mathrm{Co}_{3} \mathrm{O}_{4}$ nano-micro arrays featuring superior activity as cathode in a flexible and rechargeable zinc-air battery. Adv. Sci. 6, 1802243 (2019). https://doi.org/10.1002/advs.20180 2243

156. Y.-N. Chen, Y. Guo, H. Cui, Z. Xie, X. Zhang et al., Bifunctional electrocatalysts of MOF-derived Co-N/C on bamboo-like $\mathrm{MnO}$ nanowires for high-performance liquid- and solid-state Zn-air batteries. J. Mater. Chem. A 6, 9716-9722 (2018). https://doi.org/10.1039/c8ta01859f

157. X.F. Lu, Y. Chen, S. Wang, S. Gao, X.W.D. Lou, Interfacing manganese oxide and cobalt in porous graphitic carbon polyhedrons boosts oxygen electrocatalysis for $\mathrm{Zn}$-air batteries. Adv. Mater. 31, 1902339 (2019). https://doi.org/10.1002/ adma.201902339

158. H. Zou, B. He, P. Kuang, J. Yu, K. Fan, Metal-organic framework-derived nickel-cobalt sulfide on ultrathin Mxene nanosheets for electrocatalytic oxygen evolution. ACS Appl. Mater. Interfaces 10, 22311-22319 (2018). https://doi.org/ 10.1021/acsami.8b06272

159. S. Liu, X. Zhang, G. Wang, Y. Zhang, H. Zhang, Highefficiency $\mathrm{Co} / \mathrm{Co}_{\mathrm{x}} \mathrm{S}_{\mathrm{y}} @ \mathrm{~S}, \mathrm{~N}$-codoped porous carbon electrocatalysts fabricated from controllably grown sulfur- and nitrogen-including cobalt-based MOFs for rechargeable zincair batteries. ACS Appl. Mater. Interfaces 9, 34269-34278 (2017). https://doi.org/10.1021/acsami.7b11101

160. J.-Y. Zhao, R. Wang, S. Wang, Y.-R. Lv, H. Xu et al., Metalorganic framework-derived $\mathrm{Co}_{9} \mathrm{~S}_{8}$ embedded in $\mathrm{N}, \mathrm{O}$ and $\mathrm{S}$-tridoped carbon nanomaterials as an efficient oxygen bifunctional electrocatalyst. J. Mater. Chem. A 7, 7389-7395 (2019). https://doi.org/10.1039/c8ta12116h

161. I.S. Amiinu, Z. Pu, X. Liu, K.A. Owusu, H.G.R. Monestel et al., Multifunctional Mo-N/C@ $\mathrm{MoS}_{2}$ electrocatalysts for HER, OER, ORR, and Zn-air batteries. Adv. Funct. Mater. 27, 1702300 (2017). https://doi.org/10.1002/adfm.201702300

162. H. Liu, J. Guan, S. Yang, Y. Yu, R. Shao et al., Metal-organicframework-derived $\mathrm{Co}_{2} \mathrm{P}$ nanoparticle/multi-doped porous carbon as a trifunctional electrocatalyst. Adv. Mater. 32, 2003649 (2020). https://doi.org/10.1002/adma.202003649

163. D.D. Babu, Y. Huang, G. Anandhababu, M.A. Ghausi, Y. Wang, Mixed-metal-organic framework self-template synthesis of porous hybrid oxyphosphides for efficient oxygen evolution reaction. ACS Appl. Mater. Interfaces 9, 38621-38628 (2017). https://doi.org/10.1021/acsami.7b13359

164. Y. Hao, Y. Xu, W. Liu, X. Sun, Co/CoP embedded in a hairy nitrogen-doped carbon polyhedron as an advanced tri-functional electrocatalyst. Mater. Horizons 5, 108-115 (2018). https://doi.org/10.1039/c7mh00706j 
165. Y.-S. Wei, M. Zhang, M. Kitta, Z. Liu, S. Horike et al., A single-crystal open-capsule metal-organic framework. J. Am. Chem. Soc. 141, 7906-7916 (2019). https://doi.org/10.1021/ jacs.9b02417

166. C.-L. Zhang, J.-T. Liu, H. Li, L. Qin, F.-H. Cao et al., The controlled synthesis of $\mathrm{Fe}_{3} \mathrm{C} / \mathrm{Co} / \mathrm{N}$-doped hierarchically structured carbon nanotubes for enhanced electrocatalysis. Appl. Catal. B-Environ. 261, 118224 (2020). https://doi.org/ 10.1016/j.apcatb.2019.118224

167. J.-T. Liu, Y. Xie, Q. Gao, F.-H. Cao, L. Qin et al., 1D MOFderived $\mathrm{N}$-doped porous carbon nanofibers encapsulated with $\mathrm{Fe}_{3} \mathrm{C}$ nanoparticles for efficient bifunctional electrocatalysis. Eur. J. Inorg. Chem. 2020, 581-589 (2020). https://doi.org/ 10.1002/ejic.201901244

168. G. Chen, T. Wang, P. Liu, Z. Liao, H. Zhong et al., Promoted oxygen reduction kinetics on nitrogen-doped hierarchically porous carbon by engineering proton-feeding centers. Energy Environ. Sci. 13, 2849-2855 (2020). https://doi.org/10.1039/ d0ee01613f

169. F. Meng, H. Zhong, D. Bao, J. Yan, X. Zhang, In situ coupling of strung $\mathrm{Co}_{4} \mathrm{~N}$ and intertwined $\mathrm{N}-\mathrm{C}$ fibers toward freestanding bifunctional cathode for robust, efficient, and flexible Zn-air batteries. J. Am. Chem. Soc. 138, 10226-10231 (2016). https://doi.org/10.1021/jacs.6b05046

170. Y. Wang, Q. Cao, C. Guan, C. Cheng, Recent advances on self-supported arrayed bifunctional oxygen electrocatalysts for flexible solid-state Zn-air batteries. Small 16, 2002902 (2020). https://doi.org/10.1002/smll.202002902

171. C. Guan, A. Sumboja, W. Zang, Y. Qian, H. Zhang et al., Decorating $\mathrm{Co} / \mathrm{CoN}_{\mathrm{x}}$ nanoparticles in nitrogen-doped carbon nanoarrays for flexible and rechargeable zinc-air batteries. Energy Stor. Mater. 16, 243-250 (2019). https://doi.org/10. 1016/j.ensm.2018.06.001

172. Q. Xu, H. Jiang, Y. Li, D. Liang, Y. Hu et al., In-situ enriching active sites on co-doped $\mathrm{Fe}-\mathrm{Co}_{4} \mathrm{~N} @ \mathrm{~N}-\mathrm{C}$ nanosheet array as air cathode for flexible rechargeable $\mathrm{Zn}$-air batteries. Appl. Catal. B-Environ. 256, 117893 (2019). https://doi.org/10. 1016/j.apcatb.2019.117893

173. T. Meng, J. Qin, S. Wang, D. Zhao, B. Mao et al., In situ coupling of $\mathrm{Co}_{0.85} \mathrm{Se}$ and $\mathrm{N}$-doped carbon via one-step selenization of metal-organic frameworks as a trifunctional catalyst for overall water splitting and Zn-air batteries. J. Mater. Chem. A 5, 7001-7014 (2017). https://doi.org/10.1039/c7ta0 $1453 \mathrm{~h}$

174. H. Wu, J. Wang, J. Yan, Z. Wu, W. Jin, MOF-derived twodimensional $\mathrm{N}$-doped carbon nanosheets coupled with
$\mathrm{Co}-\mathrm{Fe}-\mathrm{P}-\mathrm{Se}$ as efficient bifunctional OER/ORR catalysts. Nanoscale 11, 20144-20150 (2019). https://doi.org/10.1039/ c9nr05744g

175. Q. Wang, L. Shang, R. Shi, X. Zhang, Y. Zhao et al., NiFe layered double hydroxide nanoparticles on $\mathrm{Co}, \mathrm{N}$-codoped carbon nanoframes as efficient bifunctional catalysts for rechargeable zinc-air batteries. Adv. Energy Mater. 7, 1700467 (2017). https://doi.org/10.1002/aenm.201700467

176. Y. Qian, T. An, E. Sarnello, Z. Liu, T. Li et al., Janus electrocatalysts containing MOF-derived carbon networks and $\mathrm{NiFe}-\mathrm{LDH}$ nanoplates for rechargeable zinc-air batteries. ACS Appl. Energy Mater. 2, 1784-1792 (2019). https://doi. org/10.1021/acsaem.8b01923

177. D. Chen, X. Chen, Z. Cui, G. Li, B. Han et al., Dual-activesite hierarchical architecture containing NiFe-LDH and ZIF-derived carbon-based framework composite as efficient bifunctional oxygen electrocatalysts for durable rechargeable Zn-air batteries. Chem. Eng. J. 399, 125718 (2020). https:// doi.org/10.1016/j.cej.2020.125718

178. J.P. Guerrette, S.J. Percival, B. Zhang, Fluorescence coupling for direct imaging of electrocatalytic heterogeneity. J. Am. Chem. Soc. 135, 855-861 (2013). https://doi.org/10.1021/ ja310401b

179. J.A. Haber, Y. Cai, S. Jung, C. Xiang, S. Mitrovic et al., Discovering Ce-rich oxygen evolution catalysts, from high throughput screening to water electrolysis. Energy Environ. Sci. 7, 682-688 (2014). https://doi.org/10.1039/c3ee43683g

180. Z.-Q. Cao, M.-Z. Wu, H.-B. Hu, G.-J. Liang, C.-Y. Zhi, Monodisperse $\mathrm{Co}_{9} \mathrm{~S}_{8}$ nanoparticles in situ embedded within $\mathrm{N}, \mathrm{S}$-codoped honeycomb-structured porous carbon for bifunctional oxygen electrocatalyst in a rechargeable $\mathrm{Zn}$-air battery. NPG Asia Mater. 10, 670-684 (2018). https://doi.org/ 10.1038/s41427-018-0063-0

181. J.-C. Dong, X.-G. Zhang, V. Briega-Martos, X. Jin, J. Yang et al., In situ Raman spectroscopic evidence for oxygen reduction reaction intermediates at platinum single-crystal surfaces. Nat. Energy 4, 60-67 (2018). https://doi.org/10. 1038/s41560-018-0292-z

182. S. Zhao, C. Tan, C.-T. He, P. An, F. Xie et al., Structural transformation of highly active metal-organic framework electrocatalysts during the oxygen evolution reaction. Nat. Energy 5, 881-890 (2020). https://doi.org/10.1038/ s41560-020-00709-1 